\title{
Forschungen zur politischen Rolle der stadtrömischen Plebs in der Kaiserzeit*
}

\author{
Katja Kröss
}

Die Realencyclopädie lässt in einem Artikel von Wilhelm Hoffmann aus dem Jahr 1951 die politische Geschichte der Plebs mit der Republik enden: „Die Kaiserzeit brachte den Abschluß in der Geschichte der P[lebs]. Sie schied nun scharf zwischen den regierenden Kreisen, in Rom dem ordo equester und senatorius [...] auf der einen, der P[lebs] auf der anderen Seite. [...] Diese P[lebs] aber hatte keinen Einfluß mehr. [...] Unter P[lebs] versteht man jetzt den Teil der Bevölkerung, der an dem vom Staat zu verbilligten Preisen oder umsonst abgegebenen Getreide beteiligt war." ${ }^{11}$ Dieses Urteil, das an Iuvenals berühmtes Diktum des nur an panis et circenses interessierten Volkes anlehnt, spiegelt den damaligen Zeitgeist wider. ${ }^{2}$ Er und bei manchen Wissenschaftlern wohl auch der elitäre Hintergrund erlaubten es nicht, das Volk, das sich ihnen als unüberschaubare Masse, als ,Pöbel“ und ,Mob“ - plastischer: als ,verlumptes, bald bettelndes, bald tobendes und drohendes Proletariat" - darstellte, als wesentlichen Bestandteil von Gesellschaft und Politik wahrzunehmen. ${ }^{3}$ So hatten sich zwar ab Ende des 19. Jahrhunderts vereinzelt Studien mit der stadtrömischen Plebs der Kaiserzeit befasst, politische Aspekte blieben jedoch weitgehend ausgeklammert: Das Privatleben des ,einfachen' Volkes (dann allerdings nicht nur der Hauptstadt) sowie die negativ interpretierten Aspekte Brot und Spiele standen im Mittelpunkt. ${ }^{4}$ Selbst in Arbeiten wie der Sittengeschichte Ludwig Friedländers, der die Plebs als „den dritten Stand “5 bezeichnet und ihrer Darstellung weit mehr Platz einräumt als derjenigen der Senatoren und Ritter, ihr Aktionspotential zuspricht und gar die These von den Schauspielen als Ersatz für die Volksversammlungen aufstellt, findet eine Reflexion über ihre politische Bedeutung nicht $\operatorname{statt}^{6}$ - oder aber diese wird, wie in Ulrich

\footnotetext{
* Für kritische Lektüre bin ich wie immer Agnes Luk zu großem Dank verpflichtet.

${ }^{1}$ Hoffmann 1951, 102; die Arbeit von van Berchem 1939 berücksichtigt er nicht. Vgl. Hoffmann 1938, 83: „Einst hoben sich nur die patricii von der plebs ab, später traten als Gegensatzpaar dazu die nobiles, die Senatoren, die equites, bis schließlich zu Beginn der Kaiserzeit das Wort herabsank zu der Bedeutung Pöbel, von dem sich alles distanziert, was einen eigenen Wert besitzt oder Anspruch auf eigene Geltung erhebt.“

${ }^{2}$ Iuv. 10,77-81; vgl. Fronto p.213,11f. v.d.H. S. etwa auch RostovtZEFF 1957² (1926), 81: „The hundreds of thousands of Roman citizens who lived in Rome cared little for political rights. They readily acquiesced in the gradual reduction of the popular assembly under Augustus to a pure formality, they offered no protest when Tiberius suppressed even this formality, but they insisted on their right, acquired during the civil war, to be fed and amused by the government. None of the emperors, not even Caesar or Augustus, dared to encroach on this sacred right of the Roman proletariat.“ VAN BERCHEM 1939, 177 durchbricht diesen Topos in Bezug auf das Brot als Erster, indem er darauf hinweist, dass die Getreideverteilung nicht etwa, wie von MOMMSEN 18827, 506 behauptet, seit Caesar „Armenversorgung“, sondern eine politische Institution gewesen sei. Dennoch begegnen derartige Vorurteile selbst noch in jüngeren Publikationen: s. etwa Arena 2007, 28; Groot 2008, 307f. - Vgl. selbst die Beurteilung der spätrepublikanischen Plebs etwa durch SYME 1939, 476 (,no role in government, no role in history").

${ }^{3}$ CAUER 1899, 698; vgl. noch die sehr ähnliche Formulierung von ALFÖLDY 2011 ${ }^{4}$, 183, wonach „die breite Schicht der parasitären Getreideempfänger stets ein ,Lumpenproletariat" darstellte.“ Eine Ausnahme bildet ABBOTт 1911, der seine Studie über verschiedene Aspekte des Alltagslebens des einfachen Volkes mit dem Satz begründet: „We are interested in the common people of Rome because they made the Roman Empire what it was“ (vii).

${ }^{4}$ Etwa Cauer 1899, 686-702; ABBott 1911; MAXey 1938 (Berufe); CARCOPINO 19924 (1939). Nicht nur, aber auch die Plebs betreffen FrIEDLÄNDER $1922^{10}$ (1861-1871); MARQUARDT $1886^{2}$ (1864).

${ }^{5}$ FRIEDLÄNDER $1922^{10}$ I, 159; zu den drei Ständen 104-239; zum „,dritten Stand“ 159-239. S. in jüngerer Zeit noch etwa HuRley 1993, 39 zu Suet. Cal. 14,1; FraschetTi 2005², XIII und passim; zur Problematik jedoch bereits HELLEGOUARC'H $1972^{2}$, 506f.

${ }^{6}$ FrIEDLÄNDER $1922^{10}$ II, 3-9; 117f.; 145-147. Vgl. GAGÉ 1971², 124-129 und CARCOPINO $1992^{4}$, die über eine deskriptive Darstellung der stadtrömischen Plebs nicht hinauskommen.
} 
Kahrstedts Kulturgeschichte der römischen Kaiserzeit, explizit als nicht vorhanden abgetan. ${ }^{7}$

Dass Jürgen Deininger noch 1979 in einem Aufsatz, der die Darstellung der politischen Rolle der stadtrömischen Plebs durch Tacitus untersucht, moniert, dass dieser antike Autor der Plebs mehr Raum gewidmet habe als viele moderne Darstellungen, ist jedoch nicht berechtigt. ${ }^{8}$ Mitte der Sechziger war das Interesse am stadtrömischen Volk und seiner politischen Bedeutung erwacht und hatte hauptsächlich im angelsächsischen und deutschen Sprachraum zu einer ersten Forschungswelle geführt, die bis Ende der Siebziger andauern sollte: Zvi Yavetz publizierte 1969 seine bahnbrechende Arbeit Plebs and Princeps; zeitgleich erschien der Beitrag des ungarischen Althistorikers István Hahn Zur politischen Rolle der stadtrömischen Plebs unter dem Principat. Wenn auch auf den Raum des Theaters beschränkt, unterstrich außerdem Traugott Bollinger, ebenfalls im selben Jahr, in seiner Studie Theatralis licentia. Die Publikumsdemonstrationen an den öffentlichen Spielen im Rom der früheren Kaiserzeit und ihre Bedeutung im politischen Leben die grundsätzliche politische Aktivität der Plebs. ${ }^{9} 1971$ und 1972 folgten zwei Aufsätze aus dem angelsächsischen Raum, Thomas W. Africas Urban Violence in Imperial Rome und Barry Baldwins Rulers and Ruled at Rome A.D. 14-192; 1976 schließlich veröffentlichte Rolf Gilbert mit seiner Dissertation über Die Beziehungen zwischen Princeps und stadtrömischer Plebs im frühen Principat die erste einschlägige deutsche Monographie zum Thema. Sie bildet den Schlussstein der ersten Forschungsphase. Untersuchungszeitraum, Zielsetzung und Ergebnisse dieser ersten Publikationen zur politischen Rolle des stadtrömischen Volkes der Kaiserzeit sind zwar unterschiedlich, nichtsdestoweniger verfolgen sie ein einheitliches Ziel: die Dekonstruktion des Topos einer passiven und bedeutungslosen Plebs.

Ein erster Ansatz einer Neubewertung findet sich dabei noch vor Yavetz in einer Publikation, die sich nicht primär mit dem Volk befasst und deswegen von der Forschung diesbezüglich zunächst nicht wahrgenommen wurde: Enemies of the Roman Order von Ramsay MacMullen aus dem Jahr 1966. Im Kapitel Urban Unrest, das sich hauptsächlich auf Quellenbelege der Spätantike, vereinzelt jedoch auch auf solche der frühen und mittleren Kaiserzeit stützt, deutet er auf wenigen Seiten erstmals die politische Effektivität der Unruhen der städtischen Bevölkerungen im Allgemeinen und der römischen im Speziellen an. ${ }^{10}$ Diese sei zwar leichter anzunehmen als zu beweisen, doch belegten die Cleander-Affäre sowie die letzten Wochen der Kaiser Nero, Iulian und Maxentius, dass ,[s] [souts could clearly shake the throne itselff". ${ }^{11}$ Ungeachtet der in der Hauptstadt durch die hohe Truppenpräsenz gegebenen Kontrollmöglichkeiten sei es den Massen insbesondere in den Theatern möglich ge-

\footnotetext{
${ }^{7}$ KAHRSTEDT, Kulturgeschichte $1958^{2}$ (1944), $18 \mathrm{f}$.

${ }^{8}$ DEININGER 1979, 301f.

${ }^{9}$ Wie Friedländer (s. oben) versteht auch BOLLINGER 1969 die Spiele als „Ersatz der Volksversammlung“ (72) mit dem Unterschied, dass das Volk in diesem Rahmen die Initiative habe ergreifen können, die entsprechenden Äußerungen jedoch von „völlige[r] rechtliche[r] Bedeutungslosigkeit“ (73) gekennzeichnet gewesen seien. Ihr Gewicht habe darin bestanden, dass ,,[d]ie ,Institution“ der Publikumskundgebungen [...] nun einmal da war und ohne weiteres nicht aus der Welt geschafft werden konnte - ganz abgesehen davon, dass sie dem Kaiser selbst oft vorteilhaft erschien und daher willkommen war [...]. [I]n ihrem unbestimmten und schwer fassbaren Verhältnis zu Staat und Politik entspricht sie dem Principat, einer Herrschaftsform, deren wesentlichste Züge sich durch keine staatsrechtlichen Definitionen ausdrücken lassen“" (73).

${ }^{10}$ MacMulLen 1966, 164-180, insbes. 172 mit 340f. Anm. 12 und 178f. Eine systematische Erfassung der Belege erachtete er offensichtlich nicht für wichtig: s. etwa 344 Anm. 19.

${ }^{11}$ EBD., 172. Quellenbelege, die insbesondere in Bezug auf Nero sehr aufschlussreich wären, fehlen auch in der Anmerkung (341 Anm. 13).
} 
wesen, über - teils organisierte - Unruhen einen derartigen Druck auszuüben, dass die Kaiser hätten einlenken müssen. Den Grund sieht MacMullen, wenngleich er die Verbindung nicht explizit herstellt, offensichtlich in einer systemimmanenten Störanfälligkeit: „An authority resting on demonstrations must in logic succumb to them, too [...] - all respected by their equals because so clamorously applauded by their inferiors, and in danger of being dismissed if their popularity diminished." ${ }^{12}$ Einem grundsätzlichen Problem seiner Arbeit, nämlich der teils unsystematischen Vermischung von kaiserzeitlichen und spätantiken Quellen, ist es freilich geschuldet, dass eine exakte chronologische Zuordnung dieser wie anderer Thesen und damit ihre Aussagekraft für die Kaiserzeit erschwert werden. Trotz der Neubewertung der politischen Positionierung der kaiserzeitlichen Plebs konnte MacMullen schließlich deren pejorative Grundbeurteilung nicht überwinden: Auch für ihn war sie immer noch ein „mob“ und setzte sich aus ,the most ungovernable elements of the population“13 $\mathrm{zu}$ sammen.

Das Verdienst, die stadtrömische Plebs der Kaiserzeit in moralischen wie in politischen Termini rehabilitiert zu haben, kommt damit in der Tat erst Zvi Yavetz zu. ${ }^{14}$ Plebs and Princeps, die erste eigenständige und zugleich umfassendste Studie zum kaiserzeitlichen Volk, gilt auch beinahe fünfzig Jahre nach ihrem Erscheinen immer noch als das Standardwerk zum Thema, obwohl Yavetz lediglich den Zeitraum der iulisch-claudischen Dynastie untersucht und dabei den Fokus auf Caesar als Wegbereiter des neuen Systems und Augustus als ersten Princeps legt. Die Veränderungen unter dessen Nachfolgern seien nicht fundamental, weshalb er diesen nur ein kurzes Kapitel widmet. ${ }^{15}$

Mit Yavetz werden erstmals zwei essentielle Fragen gestellt: diejenige nach den Aktivitäten der Plebs und diejenige nach ihrem Platz in der ,Innenpolitik' Roms. Die recht kurze Beantwortung der ersten führt zu einer lange überfälligen Kategorisierung der Arten von (Re-)Aktionen (nicht-gewalttätige, gewalttätige sowie, als gesonderte Kategorie, die Reaktionen in den Spielstätten) und der Beweggründe, die dazu führten (ökonomische Gründe, Zwischenfälle im Theater, Verstöße gegen die elementare Gerechtigkeit, politischer Hintergrund). ${ }^{16}$ Auch wenn dieses Schema ergänzt und verfeinert werden kann, ${ }^{17}$ wird damit das vorherige ,panis-et-circenses-Korsett ${ }^{6}$ durchbrochen. ${ }^{18}$ Nicht ganz so befriedigend fällt die Antwort auf die zugegebenermaBen diffizilere Frage nach der politischen Bedeutung der stadtrömischen Plebs im neuen System aus, die Yavetz im Epilog zu geben versucht. Das liegt weniger daran, dass er sich nach eigener Aussage dazu gar nicht imstande sieht, sondern daran, dass

\footnotetext{
12 EBD., 179.

${ }^{13}$ EBD., 172.

${ }^{14}$ Yavetz' Interesse hatte bereits seit Beginn seiner wissenschaftlichen Laufbahn dem Volk, zunächst allerdings demjenigen der ausgehenden Republik, und dessen Neubewertung gegolten. In seiner unpublizierten Dissertation beschäftigte er sich mit der Haltung der Plebs gegenüber Steuerabschaffungen; es folgten Aufsätze etwa zu den Lebensbedingungen der Plebs (YAVETZ 1958), zur Bedeutung der kaiserlichen levitas (DERS. 1965a) und der vermeintlichen plebs sordida (DERS. 1965b).

${ }^{15}$ YAVETZ 1969a (ND 1988 mit neuem Vorwort), 38: ,[F]or while the ,tone“ varied from time to time, the ,music continued unchanged.“ $\mathrm{Zu}$ den Kaisern Tiberius bis Nero s. EBD., 103-120. - Mit einem Aufsatz zur Plebs in der Zeit von Vespasian bis Traian (DERS. 1987) setzte er seine Geschichte des stadtrömischen Volkes fort, während er den im Neudruck von Plebs and Princeps (xvi) angekündigten Beitrag zur Epoche der Severer schuldig blieb. Sein Aufsatz zu Vitellius und der Plebs (DERS. 1969b) kann hingegen nur bedingt als Fortsetzung angesehen werden, da er sich mehr mit der Konstruktion der Herrschaft des Vitellius als mit dem Verhalten der Plebs befasst.

16 YAVETZ 1969a, 9-37.

${ }^{17}$ S. etwa FLAIG 1992, 61-66 mit einer erweiterten Liste von ,politisierbaren` Themen.

${ }^{18}$ S. zeitgleich, jedoch ohne politische Implikationen, BALSDON 1969a, 267-269; vgl. bereits VAN BERCHEM 1939, 177 (wie Anm. 2).
} 
er sehr wohl eine präzise politische Funktion der Plebs nennt, die indes einer argumentativen Untermauerung sowohl im chronologischen Hauptteil als auch im systematischen Epilog entbehrt. Zwar sei die Plebs nie politisch dominant gewesen - an ihren Aktionen macht Yavetz ihre Bedeutung somit nicht fest -, doch belegten die Bemühungen der Principes um sie, dass sie kein ,entirely negligible factor“ ${ }^{19}{ }^{2}$ keine „Social class that lacks all political significance“ ${ }^{\text {(20 }}$ gewesen sei: Die Principes hätten die Zustimmung des Volkes, eine positive öffentliche Meinung gebraucht. Ihnen sei klar gewesen, dass Rom zwar unmöglich nur mit dessen Hilfe, aber auch schwerlich gegen dessen Wünsche zu regieren sei. Welche politischen Konsequenzen ein Handeln gegen die Interessen der Plebs hatte, tritt allerdings aus dem Text nicht klar hervor.

Noch weniger kann Yavetz seine daran anschließende Behauptung belegen, dass die stadtrömische Plebs aufgrund ihrer (zuvor nur vage umschriebenen) Bedeutung vom Kaiser als Abschreckmittel gegen den Senat und sogar gegen das Heer habe instrumentalisiert werden können. ${ }^{21}$ In der ,Fortsetzung ' von Plebs and Princeps, dem knapp zwanzig Jahre später veröffentlichten Aufsatz The Urban Plebs in the Days of the Flavians, Nerva and Trajan, in dem er diese These erneut aufgreift und dahingehend präzisiert, dass die Plebs ,,a last resort “ gegen feindliche Senatoren und rebellierende Prätorianer gewesen sei, schränkt er zwar ein, dass dies nicht häufig geschehen sei - doch auch hier nennt er nicht die Ausnahmefälle, die seine These bestätigen könnten. ${ }^{22}$ Trotz wertvoller Einzelergebnisse ist Yavetz somit hinsichtlich einer Neubewertung der politischen Rolle der stadtrömischen Plebs seiner Zielsetzung letztlich nicht gerecht geworden.

Die Reaktionen der zeitgenössischen Forschung waren dreigeteilt. Während Einzelne sich noch der alten Denkweise verpflichtet sahen und den Forschungsgegenstand per se ob der diesbezüglich angeblich aussagelosen Quellenlage gänzlich ablehnten, ${ }^{23}$ zeigten sich andere, vor allem von italienischer und französischer Seite, mehr oder weniger vorbehaltlos begeistert. ${ }^{24}$ Ihnen folgt meist die spätere, sich nicht zentral der Plebs widmende Forschung, die sich in Fragen zu derselben kritiklos auf Yavetz beruft. Ein bedeutender Teil der zeitgenössischen Forschung jedoch äußerte sich sehr wohl skeptisch. „[T] emerge as a force of serious importance. And, despite Yavetz's endeavors, the dominant impression is still one of a fickle and selfish mob", urteilt etwa Erich S. Gruen in seiner Rezension und fasst damit die zentralen Vorbehalte der kritischen Stimmen zusammen: Die Intention der Studie, die gesellschaftliche und politische Aufwertung der kaiserzeitlichen Plebs, sahen sie nicht als erfüllt an. ${ }^{25}$ Das Verdienst von Yavetz liegt auch für sie darin begründet, dass dieser eine - von vielen vorher als solche zweifelsohne gar nicht wahrgenommene - Forschungslücke aufdeckte. ${ }^{26}$ Das größte

\footnotetext{
${ }^{19}$ YAVETZ 1969a, 132.

${ }^{20}$ EBD., 135.

${ }^{21}$ EBD., 136.

${ }^{22}$ EBD.; DERS. 1987, 181.

${ }^{23}$ So BALSDON 1969b, 780; Defosse 1969; FreZZA 1969; Jones 1971.

${ }^{24}$ GabBa 1969; JAL 1969; Bernardi 1970; BADIAN 1970; PetiT 1970; Richard 1970. Vgl. mit ähnlichen Ergebnissen wie Yavetz, jedoch ohne eine etwaige Abhängigkeit offenzulegen, GAVAZZI 1977.

${ }^{25}$ Gruen 1970, 487. Vgl. Bandelli 1969; BleiCKen 1998 (1970); WiCKERT 1970; HellegouarC'H 1971; JONES 1971; OOST 1971, 137f.; SCARBOROUGH 1971; DIESNER 1973.

${ }^{26}$ Anders nur WICKERT 1970, 653, der lapidar behauptet, „,daß wir das meiste schon gewußt haben, im Kleinen und im Größeren." - Obwohl die großen Handbücher wie die Cambridge Ancient History oder die Storia di Roma die kaiserzeitliche Plebs immer noch stiefmütterlich behandeln und ihr etwa anders als den Oberschichten keinen eigenen Beitrag widmen (immerhin einige Seiten behält ihr PURCell 1996, 796-799; 805-809 vor), ist zu beobach-
} 
Manko von Yavetz' Arbeit jedoch, die eigenartige Blässe, die die Plebs hier weiterhin charakterisiert und auf die sich Gruen wohl mit dem immer noch vorherrschenden Eindruck eines „fickle and selfish mob“ bezieht, wusste nur einer in konkrete Worte zu formulieren: „[D]er Titel führt irre, ,Princeps and Plebs“ sollte er lauten. Die plebs ist hier Objekt, Subjekt dagegen ist der princeps; man könnte fast sagen, die plebs gibt nicht viel mehr ab als die Kulisse, vor der die Haltung und die Handlungsweise des princeps sich darstellen“, so Lothar Wickert in einem vernichtenden Urteil der Arbeit. $^{27}$

In Bezug auf die nachfolgende Forschung zur politischen Rolle der stadtrömischen Plebs gebührt den Kritikern von Yavetz' Plebs and Princeps selbst ein großes Verdienst. Sie legten von diesem nicht befriedigend beantwortete sowie neue Fragen offen, in deren Mittelpunkt die Vernetzung von Plebs und Oberschicht mit den dazugehörigen Implikationen - Beeinflussung, Lenkung, Manipulation ${ }^{28}$ - steht, die künftige Studien zur stadtrömischen Plebs der Kaiserzeit zu beantworten hätten. ${ }^{29}$ Dem wurde seither freilich nur teilweise entsprochen.

Im zeitgleich mit Yavetz' Plebs and Princeps 1969 erschienenen knappen Aufsatz Zur politischen Rolle der stadtrömischen Plebs unter dem Prinzipat geht auch István Hahn von der These aus, dass die moralische Dekadenz und politische Indifferenz des stadtrömischen Volkes ein literarischer Topos sei, und betrachtet den gesamten Prinzipat bis zum Beginn der Epoche der Soldatenkaiser. ${ }^{30}$ Dabei wird schnell deutlich, dass er, anders als Yavetz, die politische Bedeutung des Volkes davon abhängig macht, ob dieses greifbaren Einfluss auf politische Vorgänge ausübte: Dass die Kaiser die öffentliche Meinung der Hauptstadt immer berücksichtigt und versucht hätten, „diesen Pöbel bei guter Laune zu erhalten“31 - Hahn scheint wie MacMullen die alten Vorurteile noch nicht ganz abwerfen zu können -, ändere nichts am generellen Bild einer politischen Passivität der Plebs in den ersten beiden Jahrhunderten. Dies werde auch durch deren gelegentliche Ausbrüche nicht getrübt. Erst mit dem Fall des Cleander unter Commodus habe sich dies ,plötzlich und in erheblichem Maße“32 gewandelt und die Plebs im folgenden halben Jahrhundert noch einmal aktiven Anteil am Kampf um die Macht genommen. Die Gründe für die neuen politischen Spielräume vermutet er einerseits in der seit den Antoninen freieren Möglichkeit der Bildung von Collegia, die er als organisierende Kraft annimmt, andererseits in der seit Ende des zweiten Jahrhunderts auftretenden zeitweiligen Schwächung der kaiserlichen Macht.

Die Zweiteilung der Geschichte der stadtrömischen Plebs in eine lange passive und eine kurze aktive Phase bringt es mit sich, dass Hahns Kategorisierung der For-

ten, dass seit den siebziger Jahren des letzten Jahrhunderts Untersuchungen über sozial-politische Aspekte der Kaiserzeit vermehrt einen (meist kurzen) Abschnitt auch auf die stadtrömische Plebs verwenden: s. bspw. MILLAR $1992^{2}$ (1977), 368-375; GARNSEY/SALLER 1987, 83-88; NIPPEL 1988, 153-171; DERS. 1995, 85-98, insbes. 85-90; KIENAST 20094, 194-203. Zu Unruhen allgemein, Rom allerdings nur am Rande einbeziehend, s. etwa PEKÁRY 1987; vgl. bereits BRAUNERT 1980 (Vortrag von 1967).

${ }^{27}$ WICKERT 1970, 652. So treffend diese Feststellung ist, so ist das daran anschließende Urteil, dass die Plebs auch in ihren Aktionen „eine amorphe Masse, nicht nur in dem von uns überkommenen Geschichtsbild, sondern auch in der geschichtlichen Wirklichkeit“ bleibe, weiterhin dem alten Denkmuster verpflichtet.

${ }^{28}$ S. hierzu (zeitgleich zu Yavetz) BOLLINGER 1969, 32-44.

${ }^{29}$ BANDelli 1969, 355f.; SCARBOROugh 1971, 265f.; Diesner 1973, 89. Weitere Kritikpunkte betreffen das angebliche Bedürfnis der Plebs nach Erniedrigung der Oberschicht (BLEICKEN 1998, 794; GRUEN 1970, 488; s. aber etwa LEPPIN 1992, 63) und die Rolle der tribunicia potestas (GRUEN 1970, 488f.; vgl. bereits KUNKEL 1961, 363f.; s. jedoch immer noch LEVICK 2010, 84f.).

${ }^{30}$ HAHN 1969.

${ }^{31}$ EBD., 40.

${ }^{32}$ EBD., 41. 
men des Protests (stillschweigende Straßendemonstrationen, laute Demonstrationen in den Schauspielstätten, contiones, Straßenkrawalle) sowie der Motivationen (Versorgungsprobleme, Prestige der Stadt, Charakter und Regierungsstil der Kaiser, Hass gegen die Aristokratie) in erster Linie die kurze zweite Epoche betreffen und nur bedingt auf die ersten beiden Jahrhunderte angewandt werden können. Problematisch ist jedoch vor allem, dass Hahn - wie auch Yavetz - Fragen der Quellenproblematik nicht stellt: Beide übernehmen die von den Autoren überlieferten Ereignisse weitgehend unreflektiert.

Die Betonung der Quellenproblematik, mit der jeder, der sich mit der Geschichte der unteren Schichten befasst, konfrontiert wird, ist hingegen Hauptanliegen der Beiträge von Thomas W. Africa über The Urban Violence (1971) und Barry Baldwin über Rulers and Ruled (1972), ebenso wie die damit zusammenhängende Revision des Klischees von der unbeschäftigten, ihre Zeit in Spielstätten verbringenden Plebs. ${ }^{33}$ Dabei weisen sie nicht nur, wie Yavetz und Hahn vor ihnen, darauf hin, dass es durchaus andere Themen gegeben habe, für die sich die Plebs interessierte, sondern treten als ,Anwälte' der Plebs auf. Sie zeigen auf, dass die moralisierende Abwertung des Interesses an Brot sowie Spielen unberechtigt ist: Abgesehen davon, dass Ersteres vital gewesen sei, ${ }^{34}$ habe sich auch die Oberschicht für materielle Belange und Spiele interessiert, sodass der nur an die Plebs gerichtete Vorwurf unhaltbar sei. Während Baldwin, der die Zeit von Tiberius bis Commodus untersucht, bei der Dekonstruktion dieser Formel stehenbleibt, hebt Africa in Betrachtung der Quellen zum ersten bis vierten Jahrhundert die politischen Implikationen hervor. In der frumentatio sieht er, wie bereits Denis van Berchem ${ }^{35}$ vor ihm, ein politisches Privileg der stadtrömischen Bürgerschaft, in den Spielen hingegen die wichtigste Kommunikationsplattform zwischen ,Basis' und Kaiser: für die Plebs, um ihre Anliegen vorzutragen - Organisationen innerhalb der Plebs betrachtet er dabei durchaus als üblich ${ }^{36}$-, für den Princeps, um deren Bedürfnisse und Wünsche kennenzulernen. ${ }^{37}$

Diese unterschiedlichen Tendenzen - unpolitische vs. politische Auslegung spiegeln sich in ihrem Urteil über die grundsätzliche Bedeutung der kaiserzeitlichen Plebs wider. Baldwin ist in seinem Verdikt eindeutig. Für ihn ist, ähnlich wie für Hahn, politische Bedeutung an die Bedingung geknüpft, unmittelbar Einfluss auf die historische Entwicklung nehmen zu können: Ausschlaggebendes Kriterium ist die Gefährdung der Position des Kaisers, die durch die Plebs zu keiner Zeit gegeben gewesen sei. Die einzige Gefahr, die von ihr ausgegangen sei, sei diejenige der „organised spontaneity“: ${ }^{38} \mathrm{Da}$ sie sich leicht von Slogans habe beeinflussen lassen, habe sie von ,,individuals with ulterior motives“, 39 die er unter anderem in der Oberschicht verortet, instrumentalisiert werden können. Zu Aufruhr sei es jedoch nur höchst selten gekommen. Für Africa äußert sich die politische Rolle der Plebs einer-

\footnotetext{
${ }^{33}$ AfRICA 1971; BALDWIN 1972.

${ }^{34}$ Vgl. auch HAHN 1969, 49, der darauf hinweist, dass mit panis nicht das kostenlose, sondern das verbilligte Brot gemeint und die Forderung danach von den antiken Autoren als berechtigt anerkannt worden sei.

${ }^{35}$ VAN BERCHEM 1939, 177-179.

${ }^{36}$ Als einzigen Beleg führt AFrICA 1971, 11f. lediglich die von Dio (76[75],4,3-6 [Xiph.]) überlieferte Episode von der göttlichen Inspiration an, die die Masse im Zirkus vor dem Hintergrund des Bürgerkriegs zwischen Septimius Severus und Clodius Albinus $196 \mathrm{n}$. Chr. zu einmütigem Beifall geleitet habe, und kommentiert, dass der Autor ,not privy to the organizations among the masses“ gewesen sei.

${ }^{37}$ In seiner einige Jahre zuvor erschienenen Monographie Rome of the Caesars (1965) sind die frumentatio für Africa noch Abmilderung der Armut in der Hauptstadt und die Spiele Ablenkung von derselben; beides zusammen interpretiert er als ein Mittel zur Prävention von Unruhen.

${ }^{38}$ BALDWIN 1972, 155.

${ }^{39}$ EBD., 154.
} 
seits in der Bedeutung der öffentlichen Meinung für den Princeps, andererseits in der Verteidigung von ,,abstract principles ${ }^{\text {“40 }}$ wie Gerechtigkeit und dynastischer Legitimität, die neben Nahrungsmittelknappheiten am häufigsten zu Unruhen geführt hätten. Dass wir derartige Aktionen nur aus einigen wenigen Stellen kennen, liege weniger an der Plebs als vielmehr am fragmentarischen Zustand der Überlieferung sowie am Desinteresse und an der aristokratisch-elitären Sichtweise der Autoren. Die Bedeutung der öffentlichen Meinung, die bereits Yavetz betonte, sei hingegen hinreichend belegt. Nur „foolhardly tyrants like Caligula“441 hätten sie ignoriert. Was die Konsequenzen der Missachtung der öffentlichen Meinung anbelangt, formuliert Africa jedoch sehr viel zurückhaltender und quellenorientierter als Yavetz: Unter derartigen ,Tyrannen“ sei der Plebs nichts anderes übrig geblieben als finstere Miene zum bösen Spiel zu machen. Seien Forderungen der Plebs zu lange ignoriert worden, habe es zwar zu Ausschreitungen kommen können. Ob allerdings realpolitische Konsequenzen daraus zu resultieren vermochten, lässt auch er unbeantwortet.

Der erste deutsche Beitrag zur Diskussion, Rolf Gilberts Dissertation von 1976 über Die Beziehungen zwischen Princeps und stadtrömischer Plebs im frühen Principat, hat mit Yavetz' Arbeit zwei zentrale Punkte gemein: Wiederum nimmt Augustus wegen seines hohen exemplarischen Stellenwertes den größten Raum ein, und wiederum liegt die Konzentration weniger auf der Plebs als vielmehr auf dem Princeps. ${ }^{42}$ Gilbert untersucht die an das stadtrömische Volk adressierten propagandistischen Instrumente, wobei er insbesondere den Münzen eine hohe Bedeutung beimisst. Nur knapp führt er aus, dass das stadtrömische Volk in der frühen Herrschaftsphase des Augustus zwar eine verlässliche und wichtige Stütze beim Aufbau des Prinzipats und noch in die tagespolitischen Auseinandersetzungen involviert gewesen sei. Danach sei es jedoch in seinen politischen Möglichkeiten in den Hintergrund gedrängt worden, sodass man von einer ,faktische[n] Ohnmacht ${ }^{643}$ sprechen müsse. Die Notwendigkeit für ,Propaganda' und mithin für ein positives Bild der Principes in der Öffentlichkeit sieht er aus zwei Gründen gegeben: aufgrund des der Plebs trotzdem weiterhin innewohnenden politischen (Stör-)Potentials, das es mithilfe von Defensivmaßnahmen in Schach zu halten gegolten habe, und aufgrund der Tatsache, dass der Princeps, damit zusammenhängend, die ,demonstrative Loyalität plebiszitären Charakters ${ }^{\text {“44 }}$ benötigt habe.

Bemühungen der Kaiser für und um die Plebs, Aktionspotential, Organisation, Positionierung im neuen politischen System - diese vier zentralen Aspekte zur politischen Rolle des stadtrömischen Volkes der Kaiserzeit, die auch die späteren Arbeiten prägen, wurden in diesen ersten Auseinandersetzungen mit der Plebs in den Jahren 1969 bis 1976 mit unterschiedlicher Schwerpunktsetzung bereits angeschnitten, jedoch nicht immer zufriedenstellend bearbeitet. Weitgehend ausgeschöpft wurde das Quellenmaterial mit den Arbeiten von Yavetz und Gilbert in Bezug auf den Aspekt der Bemühungen der Kaiser um die Plebs. Die daraus resultierende Beobachtung einer politischen Bedeutung des stadtrömischen Volkes wurde hingegen noch sehr zurückhaltend und vage formuliert. Größtes Manko blieb seit Yavetz zweifelsohne, dass weniger auf die Plebs selbst als vielmehr auf den Kaiser in seiner Beziehung zur Plebs eingegangen wurde. Wurden Situationen, die die Plebs als (Re-)Akteur erlebten, un-

\footnotetext{
${ }^{40}$ AFRICA 1971, 13.

${ }^{41}$ EBD., 11.

42 GILBERT 1976.

${ }^{43}$ EBD., 276.

${ }^{44}$ EBD., 274.
} 
tersucht, begnügte man sich zumeist mit einer unkritischen Übernahme der jeweiligen Quellenberichte. Die Frage nach einer möglichen organisierenden Kraft hinter den Aktionen und dabei insbesondere nach den Interaktionen zwischen verschiedenen gesellschaftlichen Gruppierungen wiederum wurde, wo gestellt, weniger wissenschaftlich als vielmehr wie eine Glaubensfrage gehandhabt, deren Beantwortung unabhängig von (kritisch gelesenen) Belegen erfolgen kann. Die stadtrömische Plebs der Kaiserzeit bot somit weiterhin ausreichend offene Fragen für neue Untersuchungen.

Diese setzen mit einer zweiten kleinen Forschungswelle in den neunziger Jahren ein. Nun steht die Frage nach der Positionierung des Volkes im neuen politischen System des Prinzipats im Mittelpunkt: Es sind vor allem Studien, die sich mit dem politischgesellschaftlichen System der Kaiserzeit und seinem Funktionieren befassen, die bemüht sind, die Plebs in die politische Gesamtformel einzubeziehen. ${ }^{45}$ Zwei Haupttendenzen lassen sich ausmachen. Die eine Position, deren Vertreter vor allem aus dem französischen und englischen Sprachraum kommen, sieht die Bedeutung der Plebs in ihrem Status als ,Visitenkarte' des Princeps und somit in ihrer Außenwirkung begründet. Eine Auseinandersetzung mit den Aktionen des stadtrömischen Volkes und den dahinter stehenden Mechanismen sowie Interaktionen wird in diesen Arbeiten nicht als notwendig erachtet. Die andere Ausrichtung, die in Deutschland Verbreitung fand und findet, sieht die Rolle der Plebs in ihrer Funktion als legitimierende Kraft der Position des Kaisers, mithin in ihrer Innenwirkung, und betrachtet deren Aktionen hierfür zumindest etwas genauer.

Ausgangspunkt der Deutung der Plebs als ,Visitenkarte' des Princeps ist eine sehr frühe Arbeit: Paul Veynes Monographie Le pain et le cirque von 1976 über den Euergetismus in der Antike. ${ }^{46}$ Die in Brot und Spielen bestehende liberalitas der römischen Kaiser erklärt Veyne damit, dass Rom, ,la Ville par excellence““, ${ }^{47}$ den Rang eines Hofes eingenommen habe. Nicht etwa um die Plebs ruhig zu halten - seit der Einrichtung der Prätorianergarde seien Unruhen nicht mehr zu befürchten gewesen, zudem sei Rom ohnehin keine aufrührerische Stadt gewesen -, sondern weil Rom „une ,vitrine““48 der kaiserlichen Prachtentfaltung, „le théâtre de l'apparat souverain ${ }^{\text {“49 }}$ gewesen sei, hätten sich derartige Anstrengungen und Ressourcen auf die Stadt konzentriert. Das stadtrömische Volk reduziert er folglich auf Statisten, die nur eine zeremonielle Rolle zu erfüllen gehabt hätten.

Diese rein zeremonielle Funktion kritisiert Miriam T. Griffin, die das Stichwort ,Kaiserhof" fünfzehn Jahre später in ihrem Aufsatz Urbs Roma, Plebs and Princeps von 1991 aufgreift. ${ }^{50}$ Auch sie schließt eine ,(innen-)politische“ Bedeutung der Plebs aus, nachdem einerseits die Comitien bedeutungslos gewesen seien und andererseits weder der Hass der Plebs einen Kaiser habe stürzen noch deren Zuneigung ihn habe retten können. Rom als ,the showpiece of the Empire“, als ,the visible manifestation of that deterrent power" ${ }^{\text {"51 }}$ habe vielmehr Vorbildcharakter und eine Ordnungsfunktion für Provinzen und Klientelstaaten gehabt, die, anders als Rom, nicht flächendeckend rein militärisch unter Kontrolle zu halten gewesen seien. Ihren Ver-

\footnotetext{
${ }^{45} \mathrm{~S}$. außerdem die diversen Studien von Catherine Virlouvet, die sich mit dem Teilaspekt der Versorgung der Plebs befassen, dabei insbes. VIRLOUVET 1985.

${ }^{46}$ VEYNE 1976, insbes. 682-685.

${ }^{47}$ EBD., 683.

${ }^{48}$ EBD., 682.

49 EBD., 683.

${ }^{50}$ GRIFFIN 1991.

${ }^{51}$ Beide Zitate EBD., 43.
} 
tretern, die in die Urbs kamen, habe sich diese als Stadt der Weltherrscher präsentieren müssen - und dem habe die Ausstattung der Stadt und insbesondere der Spielstätten mit prächtigen Materialien ebenso gedient wie die in Toga herausgeputzten Stadtbewohner, die sich im Theater gemäß ihren Standesunterschieden in hierarchischer Ordnung präsentierten: „A shabby, starving and obviously discontented population would not have made a good advertisement for Roman rule and for that consensus universorum that the Principate claimed to command." ${ }^{52}$ Eine Statistenrolle wird der Plebs somit auch von Griffin zugeschrieben. Anders als Veyne spricht sie ihr jedoch nicht nur die Funktion eines Spiegels der kaiserlichen Pracht und Ordnung, sondern ebenso eine nach außen systemstabilisierende Wirkung zu.

Jon E. Lendon, der sich 1997 in Empire of Honour. The Art of Government in the Roman World die Frage nach dem Funktionieren des politischen Systems des Prinzipats stellt, findet die Antwort hingegen im ,great network of honouring“ ${ }^{\circ 5}$ das die ganze römische Gesellschaft durchzogen habe. Dieser universelle ,Systemkleber" Ehre habe, wie er in einem knappen Ausschnitt im Kapitel über den Kaiser erläutert, ${ }^{54}$ diesen auch an das stadtrömische Volk gebunden: Solange der Princeps vom ,constitutional sentiment ${ }^{\text {t55 }}$ habe zehren können, habe die Bedeutung der Plebs in der Funktion als Prestigeobjekt für den Princeps gelegen - im Positiven wie im Negativen. Applaus und Zustimmung hätten zum Prestige des Kaisers beigetragen, Verweigerung und zumal Protest hingegen zu Gesichtsverlust und ,public destruction of imperial honour“" geführt. ${ }^{56}$ Dass die Plebs ,,not just a beast to be placated““ ${ }^{57}$ sondern eine im prestigeerzeugenden Sinn neben Heer und Aristokratie wichtige Stütze der Herrschaft des Kaisers gewesen sei, zeige nicht zuletzt der Entschluss Kaiser Konstantins, für seine neue Hauptstadt Konstantinopel ebenfalls eine städtische Plebs zu ,rekrutieren' ${ }^{58}$ der er dieselbe Aufmerksamkeit habe zukommen lassen wie der römischen. Eine darüber hinausgehende Bedeutung der Aktionen der Plebs stellt Lendon nicht fest.

Die zweite Deutungsrichtung zur Positionierung der Plebs im politischen System der Kaiserzeit ist diejenige, die im deutschsprachigen Raum vor allem durch das Modell des sogenannten Akzeptanzsystems bekannt ist. Beinahe zeitgleich, aber unabhängig voneinander erschienen mit Egon Flaigs Den Kaiser herausfordern. Die Usurpation im römischen Reich (1992) und Julia Sünskes Thompsons Demonstrative Legitimation der Kaiserherrschaft im Epochenvergleich. Zur politischen Macht des stadtrömischen Volkes (1993) zwei Studien, die sich hinsichtlich Fragestellung und Untersuchungszeitraum zwar unterscheiden: Flaig, der den Begriff des Akzeptanzsystems prägte, ${ }^{59}$ geht es um die Neulesung des politischen Systems der Kaiserzeit mit zeitlichem Schwerpunkt auf der taciteischen Berichterstattung (14-69 n. Chr.), Süns-

\footnotetext{
${ }^{52}$ EBD. Diese Funktion wird Rom, von Griffin unerwähnt, auch in Dios Maecenas-Rede $(52,30,1)$ zugeschrieben:

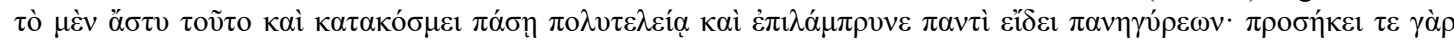

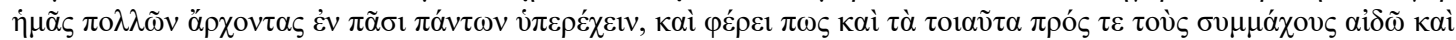

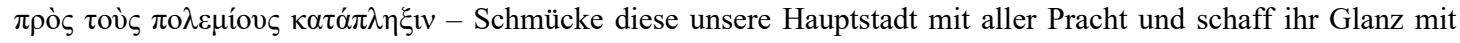
Festlichkeiten jeglicher Art! Denn es ist wohl angezeigt, daß wir, die Herren über zahlreiche Völker, alle Menschen in allen Dingen übertreffen, und Prunk solcher Art trägt auch dazu bei, unsere Bundesgenossen mit Ehrfurcht, unsere Feinde aber mit Schrecken zu erfüllen (Übersetzung: O. VEH).

${ }^{53}$ LENDON 1997, 23.

${ }^{54}$ EBD., $120-124$.

${ }^{55}$ EBD., 120.

${ }^{56}$ EBD., 121.

${ }^{57}$ EBD., 120.

${ }^{58}$ EBD.: ,Constantine's decision to recruit [...] an urban mob [...].“

${ }^{59}$ FLAIG 1992, 12.
} 
kes Thompson in ihrer kurzen Studie um die Gestaltung der politischen Macht der Plebs und deren Veränderung von der iulisch-claudischen Epoche zur Zeit zwischen 180 und 238 n. Chr. ${ }^{60}$ In Bezug auf die Plebs und ihre Bedeutung stimmen sie jedoch überein: Da das politische System der Kaiserzeit auf dem consensus universorum beruht habe und das stadtrömische Volk einer der maßgeblichen Sektoren der römischen Gesellschaft gewesen sei, sei dessen Zustimmung respektive „Teilnahme am Konsensakt ${ }^{\text {"61 }}$ ebenso konstitutiv für die Legitimität des Princeps gewesen wie diejenige der beiden anderen maßgeblichen Sektoren Senat und Heer.

Obwohl sich der Dritte im Bunde, Alfred Reese, mit seiner 2004 abgeschlossenen Dissertation Die Bürger und ihr Kaiser. Die plebs urbana zwischen Republik und Prinzipat einer machtpolitischen Einordnung der stadtrömischen Plebs der Kaiserzeit ausdrücklich verwehrt, unterscheidet er sich, trotz einiger abweichender Detailergebnisse, in der diesbezüglichen Ausrichtung kaum von derjenigen Flaigs. ${ }^{62}$ Ihm geht es wie diesem darum aufzuzeigen, dass mit der Kaiserzeit nicht etwa eine Entpolitisierung der Plebs stattgefunden habe, sondern, bedingt durch das neuartige Konsenssystem des Prinzipats, im Gegenteil von einer Politisierung erst mit der Kaiserzeit ausgegangen werden könne:63 Erst durch die Monopolisierung sämtlicher die Plebs betreffenden Bereiche durch Augustus, mithin die gemeinsame Orientierung der Plebs auf einen Einzelnen statt auf viele Aristokraten, sei die Plebs von einer ,aus dem politischen Raum ausgeschlossen[en], gespalten[en] und instrumentalisierbar[en] ${ }^{\text {"64 }}$ Menge zu einer ,selbstbewußten, eigenständigen und geschlossenen Statusgruppe“ ${ }^{65}$ geworden. Als solche habe sie sich erstmals ,permanent politisch

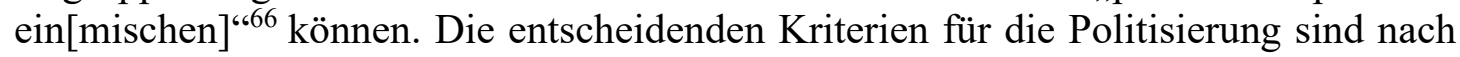
Reese somit die Geschlossenheit sowie die Unmöglichkeit der Instrumentalisierung durch Mitglieder der Aristokratie.

Die Feststellung der Bedeutung des consensus universorum für den Prinzipat sowie die der öffentlichen Zustimmung (auch) seitens des stadtrömischen Volkes sind weder neu noch problematisch. Dass der consensus universorum eine zentrale Komponente des politischen Systems der Kaiserzeit war, formulierte bereits Hans U. Instinsky in einem Aufsatz von 1940 in aller Deutlichkeit, wenngleich er nicht explizit Bezug auf die Teilnehmer am Konsensakt nahm. ${ }^{67}$ Eine explizite Einbeziehung der stadtrömischen Plebs in den consensus erfolgte jedoch durch Gilbert und später Griffin. ${ }^{68}$ Im Sinne eines Anteils der Plebs am consensus universorum ist ebenso die öffentliche Meinung zu verstehen, deren Bedeutung Yavetz und mit ihm Africa hervorhoben. ${ }^{69}$ Die demonstrative Legitimation der Principes durch die Plebs wiederum nahmen MacMullen und, wie bereits zitiert, Gilbert quasi wortwörtlich voraus: Von „[a]n authority resting on demonstration“, aus der sich die Bemühungen der Kaiser um das stadtrömische Volk erklärten, spricht der eine, von der ,demonstrative[n] Lo-

\footnotetext{
${ }^{60}$ Vgl. zur Übereinstimmung SÜNSKES THOMPSON 1993, 3 Anm. 5. Die Interpretationen des gesamtpolitischen Systems weichen hingegen teils voneinander $\mathrm{ab}$.

${ }^{61}$ FLAig 1992, 196.

${ }^{62}$ REESE 2004, s. insbes. $158 \mathrm{f}$.

${ }^{63}$ EBD., insbes. 74f. mit Anm. 224; 152-159; FLAIG 1992, 38-59.

${ }^{64}$ REESE 2004, 152.

${ }^{65}$ EBD., 11.

${ }^{66}$ EBD., 73.

${ }^{67}$ INSTINSKY 1940. Er baut seinerseits auf Vorgedanken von HoHL 1933, insbes. 109, WeBER 1936, insbes. 174, und VON PREMERSTEIN 1937, insbes. 63, auf.

${ }^{68}$ GILBERT 1976, 146f. (in der Dreierkonstellation mit Senat und Ritterstand); GRIFFIN 1991, 43. Vgl. bspw. auch MARTIN 1991, 88f.; WITSCHEL 2006, 87f.

${ }^{69}$ YAVETZ 1969a, 133-139; vgl. DENS. 1979, insbes. 215-228; AFRICA 1971, 11.
} 
yalität plebiszitären Charakters" der andere. ${ }^{70}$ Angesichts einer bis dahin gegenüber der Plebs weiterhin oftmals negativen Grundhaltung der Forschung, die sich nicht primär mit dieser befasste, gebührt insbesondere Flaig und Sünskes Thompson dennoch ein mehrfaches Verdienst: Sie sind erstmals um ein umfassenderes, über die politische Formel hinausgehendes Bild der kaiserzeitlichen Plebs bemüht, sie lenkten den Blick der Forschung endlich primär auf deren aktiven Part und schafften es, das stadtrömische Volk zumindest im deutschen Sprachraum als zentralen, positiv zu wertenden Bestandteil von Politik und nicht lediglich als moralisch verkommenen Störund Unruhefaktor zu etablieren.

Gleichwohl erweisen sich die drei Arbeiten in manch anderer, nicht minder bedeutender Hinsicht als revidierbar. Zunächst ist das Konzept der diversen ,Rollen“ des Kaisers, das insbesondere Flaig herausarbeitet, zwar von eminenter Bedeutung für das Verständnis des Prinzipats. In der Reduzierung dieser kommunikativen Beziehung

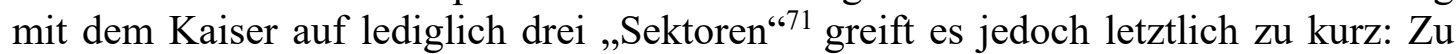
bevorzugen ist ein sehr viel differenzierterer, weitere Gruppierungen wie Provinziale einbeziehender Ansatz, wie ihn Ronald R.R. Smith 1987 für das Konzept von Ideologie skizziert und Christian Witschel 2006 in einem Beitrag zu den ,verrückten“ Kaisern systematisch dargelegt hat. ${ }^{72}$ Problematisch ist dann die politische Gewichtung der Plebs in der den consensus konstituierenden Dreierkonstellation. So bestätigt Flaig die bereits von Yavetz festgestellte unsichere politische Standortbestimmung der Plebs - schließlich habe sie nie militärisch eingesetzt werden können. Im selben Atemzug stellt er sie aber mit Senat und Heer gleich, wenn er schreibt, dass diese Unsicherheit ebenso auf die beiden letztgenannten Gruppierungen zutreffe. ${ }^{73}$ Die Senatsaristokratie wird in ihrer politischen Bedeutung der Plebs sogar untergeordnet: Nicht selten habe die Meinung des stadtrömischen Volkes eine höhere politische Intensität als diejenige der Aristokratie erreicht, vielleicht sei es sogar politisierter gewesen als der Senat, der im Gegensatz zur Plebs im dritten Jahrhundert als politische Kraft erloschen sei - der Unterschied zwischen politischer Institution und gesellschaftlicher Gruppierung wird hierbei nivelliert. ${ }^{74}$ In einem späteren Beitrag spitzt Flaig diese These noch zu und behauptet, dass, nimmt man die Kommunikation der Beherrschten mit dem Herrscher als Kriterium von Politik, „es wohl in der Weltgeschichte kaum eine Gruppe gegeben [habe], die so politisiert war wie die stadtrömische Plebs." ${ }^{\text {"75 }}$ Dass er zu dieser frappierenden These kommen kann, ist wohl auch darauf zurückzuführen, dass seiner Arbeit zwei sich widersprechende Konzepte von ,politisch ${ }^{`} \mathrm{zu}-$ grunde liegen: Während das Akzeptanzsystem als Ganzes auf einer Definition von ,politisch“ fußt, deren zentrales Kriterium die Intensität der Kommunikation ist, nicht jedoch dasjenige der Entscheidung - einer Definition, die auf die Beurteilung des Verhältnisses von Plebs und Princeps angewandt wird -, ist das häufige Abheben auf den Verlust der Entscheidungsfähigkeit in den Abschnitten über den Senat, dem er eine Einbuße an politischer Bedeutung vorwirft, unübersehbar. ${ }^{76}$

\footnotetext{
${ }^{70}$ MacMullen 1966, 179; Gilbert 1976, 274.

${ }^{71}$ FLAIG 1992, 12 und passim.

72 SMith 1987, 88; WiTSCHEL 2006.

${ }^{73}$ Flaig 1992, 70 mit Anm. 116 zu Yavetz 1969a, 131.

${ }^{74}$ FLAig 1992, 74; vgl. 62; 67f. Vgl. zur Verzerrung der Bedeutung der Senatoren UrBan 1995, 694.

${ }^{75}$ FLAIG 1994, 119.

${ }^{76}$ Besonders deutlich wird dies EBD., 119 (Kommunikation und nicht Entscheidung als Kriterium für ,politisch') und 125-129 (Bedeutung der Entscheidung für die [Nicht-]Politisierung des Senats); vgl. DENS. 1992, 66f.; 175; 196 vs. $117-121$.
} 
Sünskes Thompson geht gar einen Schritt weiter. Die demonstrative Herrschaftslegitimierung durch die Plebs sei für Senat und Heer das entscheidende Stimmungsbarometer gewesen: „Reflex der Beliebtheit und damit Gradmesser der Unantastbarkeit eines Kaisers“. ${ }^{77}$ Zwar nie imstande, einen ihrer Favoriten auf den Thron zu bringen, habe sie unter Umständen doch Herrschaftsdauer und -ende beeinflussen können, zumal wenn sie Teile des Militärs auf ihre Seite zu ziehen vermocht habe. ${ }^{78}$ Sünskes Thompson denkt hier wohl an die familiären Verbindungen zwischen Angehörigen des Heeres und der stadtrömischen Bevölkerung, die sie im Appendix thematisiert und die bei Ersteren zu Rücksicht und Beachtung der politischen Tendenzen des Volkes (hier auch hinsichtlich der Kaiserfolge) geführt haben könnten. ${ }^{79}$ Dass die Ablehnung ein und desselben Kaisers durch (Teile von) Volk und Heer nicht in kausalem Zusammenhang stehen musste, berücksichtigt sie nicht, obwohl die Schwäche ihrer These in ihrer eigenen Arbeit durchscheint: So gesteht sie zu, dass sich trotz demonstrativer Legitimierung durch das Volk kein Kaiser ohne die Unterstützung des Heeres habe halten können ${ }^{80}$ und dass es umgekehrt „,meistens nicht von Erfolg gekrönt" gewesen sei, wenn sich ein Kaiser nach Verlust der Unterstützung des Heeres „manchmal“ an die Plebs gewandt habe. ${ }^{81}$ Das suggerierte Ausmaß an politischem Durchsetzungsvermögen der Plebs muss schließlich nicht zuletzt deshalb angezweifelt werden, weil Sünskes Thompson andeutet, dass das Volk sich seiner eigenen politischen Bedeutung als „demonstrative[...] Legitimierungsmacht" womöglich kaum bewusst gewesen sei - „ein Problem, das hier nicht weiter verfolgt werden soll.“ Selbst die Kaiser hätten dies lediglich ,je nach Intelligenz und Sensibilität wahrgenommen und bei der Herrschaftssicherung berücksichtigt" ${ }^{82}$ Wie groß konnte eine Macht sein, die von den Hauptakteuren nicht wahrgenommen wurde oder ignoriert werden konnte? Wie können die Inhaber einer solchen, geradezu unsichtbaren Macht auf eine Stufe mit Heer und Senat gestellt werden? Sowohl Flaig als auch Sünskes Thompson überbewerten den Einfluss der stadtrömischen Plebs.

Ein weiteres zentrales Problem stellt die allen drei Studien sehr ähnliche Grundannahme in der Charakterisierung der stadtrömischen Plebs als Akteur dar: die einer - für Sünskes Thompson eingeschränkt geltend - stets einmütig auftretenden, ${ }^{83}$ spontan, sprich ohne steuernde Hand handelnden Großgruppe. ${ }^{84}$ Bereits Yavetz hatte sich vehement gegen die Einmütigkeit der Plebs, die nie als ,single monolithic bo$\mathrm{dy}^{{ }^{6} 85}$ operiert habe, ausgesprochen. Solche Annahmen resultierten lediglich aus den semantischen Schwierigkeiten, vor die die antiken Autoren uns in Bezug auf die Plebs

\footnotetext{
77 SÜNSKES THOMPSON 1993, 67.

${ }^{78}$ EBD., 66-69, vgl. 57f. Als prominentes Beispiel führt sie die Geschehnisse um Macrinus an: s. KRöss (im Erscheinen) mit Verweis auf die literarische Leseweise dieser Stelle.

${ }^{79}$ SÜNSKES THOMPSON 1993, 75-83, insbes. 76 mit Anm. 195 mit der Kritik an Flaig ob der Statik seines Modells, das die Interaktionen zwischen den Sektoren nicht berücksichtige. Eine Beeinflussung der Plebs durch Angehörige des Heeres thematisiert sie trotz Betonung der „gegenseitige[n] Einflußnahme“ (76) nicht.

${ }^{80}$ EBD., 69.

${ }^{81}$ EBD., 56.

${ }^{82}$ Sämtliche Zitate: EBD., 69.

${ }^{83}$ Flaig 1992, 59f. (vgl. 68f.); ReESe 2004, 144-146 (vgl. 71; 99 Anm. 34). SÜNSKeS ThOMPSON 1993, 10-14 gesteht zwar zu, dass die unterschiedlichen Volkstermini eine soziologische Identifizierung der damit bezeichneten Gruppierungen nicht zulassen. Dennoch geht sie von einem hohen Normenkonsens innerhalb der Plebs aus und verweist, damit zusammenhängend, auf Flaigs These von den spontanen und einmütigen Reaktionen (13f. Anm. 28), ohne dies näher zu kommentieren.

${ }^{84}$ Flaig 1992, 68f.; vgl. 72-74. Mit Widersprüchen ReESE 2004, 138-143; SÜNSKES THOMPSON 1993, 10-14; vgl. 31f.; 43-45; 47f.; 54 mit Anm. 146. Eine weitere gemeinsame Grundannahme, die nicht allgemeine Zustimmung finden dürfte, ist die der ,präexistenten Entpolitisierung“ (FlAIG 1992, 46): s. REESE 2004, 11; 16; SÜNSKES THOMPSON 1993, 5 Anm. 10.

${ }^{85}$ YAVETZ 1969a, 8 (s. auch den Anhang zu den semantischen Schwierigkeiten, 141-155); vgl. DENS. 1987, 166.
} 
stellten. Von einer zumeist generalisierenden Verwendung von Volksbegriffen auf eine Bedeutungslosigkeit der Heterogenität zu schließen, kommt dem Vorgehen gleich, von den ebenfalls generalisierenden Formulierungen verschiedener Autoren zu Brot und Spielen auf die Ernährung eines arbeitsunwilligen ,Lumpenproletariats' und dessen Belustigung zu Zeitvertreib und Ablenkung zu schlussfolgern - zumal es durchaus Quellenstellen gibt, die eine Uneinigkeit der stadtrömischen Plebs nicht nur suggerieren, sondern auch explizieren. ${ }^{86}$

Ebenso verhält es sich mit der diesbezüglich wesentlichen Frage nach einer möglichen Lenkung von Aktionen, die, wie bereits hervorgehoben, in der Forschung kontrovers, aber kaum auf Basis einer sorgfältigen Quellenanalyse diskutiert wird. Wenngleich Flaig in diesem Kontext der modernen Forschung lapidar vorwirft, von „massenpsychologischer ,Erklärung““ und „Manipulationstheorie“ ${ }^{\text {"87 }}$ beeinflusst „,die Vorgänge ,gegen den schreienden Protest ${ }^{\star}$ des berichthaften Diskurses zurechtbiegen [zu] müssen" ${ }^{88}$ lassen sich für Lenkung und Manipulation sowohl explizite Hinweise als auch starke Indizien anführen. ${ }^{89}$ Das zeigen nicht zuletzt die diesbezüglichen Eingeständnisse Sünskes Thompsons und Reeses auf. Bei Reese, der sich ansonsten, wenngleich nicht im Wortlaut, Flaig anschließt, kollidiert die zentrale Aussage ,keine Möglichkeit“ für Senatoren, Klientelen zu aktivieren, mit „entsprechend wenige[n] Hinweise[n]“ in den Quellen. ${ }^{90}$ Diese benennt auch Sünskes Thompson, die die Frage nach Organisationsformen, denen Aktionen entsprungen sein könnten - zu denken sei an Collegia, Klientelen oder Circusfaktionen -, als ,,modern und [...] nicht römischer Denkweise“ entsprechend relativiert. ${ }^{91}$ Dass sie keineswegs nur die Frage als modern ansieht, ${ }^{92}$ suggeriert der Duktus ihrer Schrift, vor allem aber ihre Prämisse, dass es gar nicht darum gehe, ,wer das Volk beeinflußte, sondern um die Frage, welchen Einfluß die Plebs hatte und welche Motivationen sie zu ihren politischen Aktionen

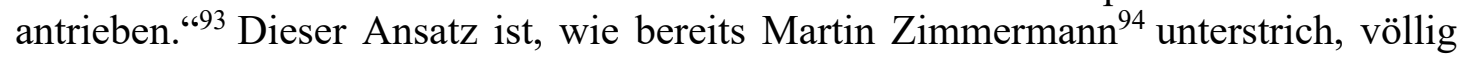
unhaltbar: Wenn eine Lenkung durch senatorische oder militärische Kreise vorgelegen haben sollte, dann sind Einfluss und Motivation durch diese Manipulation selbstverständlich (mit-)bedingt und können nicht losgelöst von ihr betrachtet werden.

\footnotetext{
${ }^{86}$ Man denke nur an die zweigeteilten Reaktionen der Plebs auf den Tod Neros, die Flaig an keiner Stelle zu erklären vermag: Tac. hist. 1,4,3; 1,16,3; 1,72,1; Suet. Nero 57,1; vgl. die Anknüpfungen Othos und Vitellius' an Nero, wie etwa von Tac. hist. 1,78,2 und D.C. 64(65),7,3 (Exc. Val.) beschrieben.

${ }^{87}$ Beide Zitate: Flaig 1992, 69.

${ }^{88}$ EBD., 72f. Anm. 127. Inwiefern sich der „Aspekt der Bezogenheit auf die aristokratischen Familien“, den Flaig EBD., 38 als einen der Gliederungsaspekte der Plebs nennt (ohne an dieser oder einer anderen Stelle weitere aufzuzählen), auswirkt, führt er nicht aus.

${ }^{89}$ S. demnächst ausführlich KRÖss 2017.

${ }^{90}$ REESE 2004, $138 \mathrm{f}$.

${ }^{91}$ SÜNSKES THOMPSON 1993, 13; Hinweise in 13 Anm. 27 (Klientelverbindungen); 10 Anm. 19 (,organisierte[...] Aktionen“ im Theater, ohne weitere Belege). In ihrer Analyse der Ereignisgeschichte nennt sie, obschon lediglich für die Epoche der Zeitgeschichte Cassius Dios, gleich fünf Episoden, in denen eine organisierende Kraft hinter den Aktionen der Plebs plausibel erscheint, und folgert daraus, dass möglicherweise „Volksdemonstrationen [...] von senatorischen Oppositionellen ermutigt, wenn nicht sogar lanciert worden sein könnten“, etwa über die Klientelverbindungen (54 Anm. 146). Bei den Episoden handelt es sich um die Stürze des Cleander (31f.), des Caracalla (43) und des Macrinus (44f.), die Kaiserwerdung Gordians III. (47 mit Anm. 135) sowie die Auseinandersetzung zwischen Maximinus Thrax und den Gordianen (47f.). Vgl. bereits DIES. 1990, 129f. und passim. S. zu den Volksaktionen der Zeitgeschichte Dios KRöss (im Erscheinen).

92 S. insbes. SÜNSKES THOMPSON 1993, 13 Anm. 27. Hier verweist sie nicht nur auf die ,wenigen Quellenhinweise“, die einen solchen Bezug dennoch herstellen, sondern hält mit Flaig ,die mißtrauische Vermutung vieler moderner Autoren für ungerechtfertigt, den politischen Aktionen der Plebs ,Drahtzieher' im Hintergrund zu unterstellen. Die Plebs hat zweifellos spontan agiert.“ Vgl. zum Vorwurf der Inkohärenz, wenngleich auf die entschiedene Ablehnung von Organisation zielend, FLAIG 1998, 137f. mit Anm. 8.

93 SÜNSKES THOMPSON 1993, 14.

${ }^{94}$ ZiMMERMANN 1999, 140 mit Anm. 119.
} 
Ein Grund für die genannten Interpretationen, die in sich und/oder zu den Belegen widersprüchlich sind, liegt im Umgang mit den Quellen. Gleichzeitig ist dies das wesentliche Problem der drei Arbeiten. Obwohl Flaig als Erster und Einziger darauf aufmerksam macht, dass die Aussagen der Autoren nicht wortwörtlich genommen werden können, ist die Umsetzung nicht gelungen. Wie bereits etliche aufmerksame Kritiker anmerkten, ${ }^{95}$ deutet er - ausgehend von einem soziologischen Ansatz, im Namen einer „praxeologischen Historie“, die einen „berichthaften“ (realhistorischen) von einem „maximischen“ (toposhaften, mit Vorurteilen belasteten) Diskurs unterscheidet - Aussagen der Autoren so um, wie sie seinem vorgefassten soziologischen Modell am besten entsprechen. ${ }^{96}$ Immer wieder werden auch Quellenpassagen verschwiegen, Parallelstellen nicht angeführt. Symptomatisch ist, dass das im systematischen Teil über die maßgeblichen Sektoren gezeichnete Bild einer politisch relevanten Plebs in der ereignishistorischen Analyse der Usurpationen keinen Widerhall findet. ${ }^{97}$

Sünskes Thompson, die bei einer ähnlichen Fragestellung wie Hahn zu ähnlichen Ergebnissen kommt - beide vergleichen die iulisch-claudische Dynastie mit dem Zeitraum 192-238 n. Chr. mit dem Resultat, dass eine Änderung der äußeren politischen Verhältnisse effektvollere Aktionen, sprich Einfluss auf die Kaiserfolge, mit sich gebracht habe -, lässt zwar eine größere Sorgfalt in der Quellenrecherche erkennen. Aufgrund der oftmals sehr unkritischen Lesung der antiken Autoren sind ihre Ergebnisse jedoch ebenfalls mit Vorsicht zu betrachten: Erst in ihrem Fazit weist sie auf eine mögliche Instrumentalisierung der Plebs durch die antiken Autoren zur Beeinflussung der zeitgenössischen Wahrnehmung und der historischen Rezeption hin $^{98}$ - ohne dass dieser essentielle Aspekt in ihre Quellenanalyse und somit in ihre Ergebnisse eingeflossen wäre. Eine häufig unkritische Übernahme der Quellenpassagen lässt sich schließlich auch der Dissertation von Reese vorwerfen. Sie weist zudem ein weiteres Problem auf: Die Arbeit, die die Plebs der ausgehenden Republik derjenigen des frühen Prinzipats gegenüberstellt - wie bei den ersten beiden Monographien zum Thema von Yavetz und Gilbert liegt der Schwerpunkt dabei auf dem Prinzipat des Augustus -, lässt letztlich in der Bemühung um eine Darstellung möglichst sämtlicher mit der Plebs in Verbindung stehender Aspekte tiefergehende Analysen vermissen und ist in ihrer Tendenz deskriptiv-zusammenfassend. ${ }^{99}$

Die neueste Untersuchung der politischen Rolle der stadtrömischen Plebs der Kaiserzeit stammt aus der Feder von Cyril Courrier. Im letzten, mit ,Depolitisée?? La plèbe et le prince d'Auguste à Domitien überschriebenen Kapitel seiner 2014 erschienenen Dissertation La plèbe de Rome et sa culture geht er als Erster ebenso ausführlich wie systematisch auf die politischen Aktivitäten des Volkes im frühen Prinzipat ein. Damit reicht seine Arbeit über Yavetz' Plebs and Princeps hinaus, obwohl sie derselben

\footnotetext{
${ }^{95}$ Baltrusch 1994; HoRstKotTe 1995, 517; Urban 1995, 692. Vgl. zu Sünskes Thompson CAMPBell 1995; ZiMMERMANN 1999, 129f. mit Anm. 58. Zu Reese liegen keine Rezensionen vor.

${ }^{96}$ S. etwa nur die exemplarische Analyse der vier Tacitus-Stellen in der methodischen Einleitung: FLAIG 1992, 1823.

${ }^{97}$ Vgl. URBAN 1995, 700, der darauf verweist, dass von einer Unterscheidung zwischen den beiden Diskursen im analysierenden Teil über die Usurpationen keine Rede mehr sein könne.

${ }^{98}$ SÜNSKES THOMPSON 1993, $73 \mathrm{f}$.

${ }^{99}$ In den vier auf die Kaiserzeit bezogenen thematischen Blöcken (Spiele, Beziehungsstrukturen, liberalitas, Beziehung Plebs und Princeps) spricht ReESE 2004, 75-151 zentrale Aspekte jeweils nur äußerst knapp an. So ist bspw. der Block „Geschenk und Privileg“ (113-132) in sieben Unterkapitel gegliedert, in denen Reese auf jeweils zwei bis fünf Seiten Aspekte wie Entwicklung der frumentatio, deren politische Bedeutung, cura annonae, congiaria, symbolische Dimension und Katastrophen abhandelt.
} 
klassischen Frage nach dem Verhältnis zwischen Volk und Kaiser verhaftet bleibt. Drei zentrale Aspekte stehen im Mittelpunkt: die Folgen des politischen Systemwechsels für die traditionellen Räume politischen Handelns des Volkes (Forum und Marsfeld), ihre Verlagerung auf Orte der direkten Begegnung zwischen diesem und dem Kaiser (insbesondere die Spielstätten) und der daraus erwachsene neue Typus politischer Macht der Plebs. Die Natur des Systems, das Courrier als „un régime officiellement républicain mais pratiquement monarchique, autrement dit, un État où la place du souverain n'était pas reconnue en tant que telle, un éventuel déficit de légitimité posait de vrais problèmes concrets “ ${ }^{\text {} 100}$ begreift, sieht er als ausschlaggebend dafür an, dass die Stütze des und die loyale Haltung gegenüber dem Princeps durch das Volk dessen fehlende ,capacités d'action [...] de faire ou défaire un empereur" ${ }^{\text {"101 }}$ aufwiegen. Damit beschreibt Courrier die politische Bedeutung der stadtrömischen Plebs in der Kaiserzeit ähnlich den Vertretern des Akzeptanzsystems in Termini der Legitimität und verneint wie diese die rhetorische Eingangsfrage nach deren vermeintlicher Entpolitisierung.

Ein zweiter zentraler Aspekt ist ihm insbesondere mit Flaig gemein: die Meinung, dass der Kaiser sich diese Stütze ,verdienen“ musste, dass ,ce consensus n'était pas gratuit et unidirectionnel." ${ }^{102}$ Die Beziehung zwischen Plebs und Princeps definiert er als „un sorte de ,contrat" social“, ${ }^{103}$ als ,le pacte civique de 1'Urbs“": ${ }^{104} \mathrm{Im}$ Austausch gegen die Legitimation habe der Kaiser für das Volk sorgen müssen - hinsichtlich der Versorgung, der Spiele, der Ausstattung der Stadt, vor allem aber hinsichtlich der Sicherung einer Lebensqualität, die Courrier im Hauptteil seiner Monographie gegen die früher landläufige Meinung der schlechten Bedingungen einer Großstadt und deren Einwohnern als ,Lumpenproletariat ${ }^{\star}$ als außergewöhnlich hoch beschreibt. ${ }^{105}$ Die Rolle, die der Princeps dabei eingenommen habe, sei die eines Vaters mit einer ,dimension clientélaire ${ }^{\text {"106 }}$ gewesen, die Beziehung, die daraus resultiert sei, persönlich (direkt, exklusiv, reziprok) ${ }^{107}$ und weder fiktiv noch gekünstelt noch metaphorisch, sondern ,une réalité quotidienne“. ${ }^{108}$ Anknüpfend an die Forschungen Frédéric Hurlets ${ }^{109}$ bringt Courrier schließlich ein seiner Ansicht nach drittes zentrales Element der neuen politischen Bedeutung des stadtrömischen Volkes ins Spiel: die Loyalität gegenüber der Familie des Kaisers, die aus der Monopolisierung der Kommunikation mit dem Volk durch den Kaiser und ,l'interaction [...] maximale“ - auch das eine These, die Courrier mit Flaig teilt - resultiert sei. ${ }^{110}$ Zusammen mit dem Princeps sei dessen Familie Garant von Sicherheit gewesen. ${ }^{111}$ Episoden rund um

\footnotetext{
${ }^{100}$ COURRIER 2014, 664.

${ }^{101}$ EBD., 663.

${ }^{102}$ EBD., 665.

${ }^{103}$ EBD., 702 mit Verweis auf MillaR 1992², 6 („The emperor was what the emperor did“) und Flaig 1992, 63 (Grund dafür, dass Schwierigkeiten in der Versorgung nicht automatisch zu feindlichen Reaktionen der Plebs gegen den Kaiser geführt hätten).

${ }^{104}$ COURRIER 2014, 742.

${ }^{105}$ EBD., 697: „De la plèbe, le princeps attendait soutien et fidélité. Il en allait (partiellement certes) de sa légitimité à gouverner. Du princeps, la plèbe exigeait protection et bienfaits. Il en allait de sa dignité, celle d'un peuple de capitale d'empire.“ Zu den Lebensbedingungen s. EBD., 27-293 mit weiterer Literatur; vgl. 735; 739-741.

${ }^{106}$ EBD., 710 gegen VEYNE 1976, 690 und mit FLAIG 1992, 79f. gegen das Verständnis dieser Beziehung als Klientel im eigentlichen Wortsinn; vgl. 665 (,[le] pluis puissant des patrons“).

107 COURRIER 2014, 699; vgl. insbes. 715-721.

${ }^{108}$ EBD., 663 (hier auch das Zitat); 697 gegen Veyne 2005, 25; 73-78; 715-721 (insbes. 719) mit Fokus auf die Antragung des pater-patriae-Titels an Augustus 2 v. Chr.

${ }^{109}$ HURLET 1997; DERS. 2009.

${ }^{110}$ COURRIER 2014, 647f.; 697f. (Zitat: 697); 721-735; vgl. FlaIG 1994, 119 (wie oben).

${ }^{111}$ Dies wird von den Quellen gestützt. Ganz deutlich wird dies etwa in zwei von Dio geschilderten Episoden der Jahre 6 n. Chr. $(55,27,3 f$.) und - sofern es sich nicht lediglich um eine Dublette handelt -8 n. Chr. $(55,33,4 f$.
} 
dynastische Nachfolgefragen sind es schließlich, auf die Courrier hauptsächlich verweist, um das Bild einer politisch durchaus proaktiven Plebs zu zeichnen. ${ }^{112}$

Obwohl Courriers innovativer Ansatz, die Möglichkeiten und den Willen der Plebs zu Aktionen von ihrer Lebenssituation her zu denken, überzeugt, gilt dies für das Ergebnis - das genannte Bild - nur eingeschränkt. ${ }^{113}$ Es scheint sich dem Duktus der Monographie zu verdanken, die eine differenzierte und vor allem positive Gesamtdarstellung der Plebs (ihrer Lebensbedingungen, ihrer ,Elite', schließlich eben ihrer politischen Rolle) zum Ziel hat. Gerade in seiner zentralen Funktion als Stütze des Princeps, als Legitimation stiftende Gruppierung sieht Courrier das Volk offensichtlich (mehrheitlich) reaktiv: ,[C]ette participation ne se limitait pas à la sanction passive d'honneurs qui pouvaient ainsi être octroyés à tel ou tel membre de la domus Augusta", heißt es im Fazit, bevor er die Beobachtung der Proaktivität der Plebs in Sachen Nachfolgeregelung anschließt: „Bien au contraire, nous avons notamment pu constater qu'elle intériorisa progressivement la notion de succession, jusqu'à pouvoir prendre elle-même position, lors des changements de règne, dans le choix du nouveau princeps “. ${ }^{114}$ Dazu passt, dass Courrier eine Erklärung, worin die konkreten (!) Probleme (,problèmes concrets“) bestehen, die dem Kaiser bei einem Legitimitätsdefizit erwuchsen, nicht gibt - zumindest nicht explizit. Sie lassen sich allerdings möglicherweise erschließen, wenn man zwei wesentliche, in unterschiedlichem Kontext gegebene Thesen kombiniert. Bezieht man nämlich die Aussage im Abschnitt über die direkte Kommunikation, wonach ,[c] [haque empereur devait assurer son propre pouvoir, puis ensuite le faire renouveler tout au long de son règne" und, so die unmittelbare Fortsetzung, ,[à] se prix était aussi le fondement de son auctoritas ${ }^{\text {"115 }}$ auf die im Fazit überraschend, da erstmalig begegnende Beobachtung, dass „la reconnaissance populaire contribuait grandement à asseoir le pouvoir du princeps et de sa famille, notamment aux yeux de 1'aristocratie“, ${ }^{116}$ dann heißt das im Ergebnis: Der Kaiser riskierte bei verweigerter Loyalitätsbezeugung des Volkes einen Verlust respektive Abstriche hinsichtlich seiner auctoritas in den Augen der Aristokratie.

Trifft diese Interpretation von Courriers Thesen zu, dann ist seine Schlussfolgerung von eminenter Bedeutung. Im Gegensatz zu den bisherigen Forschungen zur Plebs verwiese sie darauf, dass das politische Gefüge des Prinzipats sich nicht etwa auf die Beziehungen einzelner Gruppierungen zum Kaiser als alleinigem Orientierungspunkt erschöpfte. Daraus wiederum folgte, dass sich die Frage nach der politischen Bedeutung der Plebs ebenso wenig allein über das Verhältnis zwischen Plebs und Princeps beantworten lässt. Courrier freilich beschreitet im analytischen Teil genau diesen klassischen Weg. Das bedingt unter anderem, dass er mit Flaig den Aspekt möglicher Manipulation ausklammert. ${ }^{117}$ Aufgrund seiner zentralen These der proak-

[Xiph.]), die Courrier allerdings in diesem Zusammenhang außer Acht lässt (6 n. Chr.) respektive ignoriert ( $8 \mathrm{n}$. Chr.). Gemäß beiden Passagen habe Augustus komplexe Krisen jeweils mit Veranstaltungen beendet, die seinen ,Nachfolgerpool' in Szene setzten und damit den ihn überdauernden Herrschaftsanspruch des iulischen Hauses unterstrichen.

${ }^{112}$ Das wird insbesondere im Fazit (COURRIER 2014, 736) deutlich: s. unten. Vgl. EBD., 647f.; 721-735 sowie allgemein zur Proaktivität der Plebs abseits dynastischer Fragen $728 \mathrm{f}$.

113 Ähnlich bereits die Kritik von SMith 2015; vgl. VANDEVOORDE 2015, 982; anders SiLLETt 2016, 207.

${ }^{114}$ COURRIER 2014, 736.

115 EBD., 664.

${ }^{116}$ EBD., 736. Wenige Seiten später steht jedoch wieder die Konkurrenz zwischen Senat und Plebs um die Aufmerksamkeit des Princeps im Mittelpunkt (742).

${ }^{117}$ EBD., 641f. Anm. 151 mit Flaig 1992, 69 (s. oben) und der Begründung, dass die Frage nach Manipulation nicht beantwortet werden könne; vgl. 641 Anm. 149. Das führt so weit, dass er eine in den Quellen manifeste Lenkung wie 49 n. Chr. im Falle der Aufforderung an Claudius, sich mit Agrippina zu vermählen (Tac. ann. 12,5,2 mit 12,7,1), als ,attroupement spontané“ bezeichnet (729). Vgl. zur Kritik der fehlenden Frage nach Lenkung und 
tiven, selbständigen Plebs muss er diesen Aspekt freilich wohl ausklammern - und auch das gilt ähnlich für Flaig. Anders als bei diesem wird das Bewusstsein um dieses Dilemma allerdings reflektiert: allgemein im Zugeständnis, dass man diesbezüglich „plus simplement“ Flaig folgen könne, sowie als Nachtrag in der zitierten Stelle um die mögliche Oktroyierung von Akklamationen, konkret als offene Frage in einer Anmerkung zu den Demonstrationen für Octavia und gegen Poppaea 62 n. Chr. ${ }^{118}$

Vor allem aber ist die von Courrier konstruierte politische Rolle der Plebs wenig überzeugend, da auch er den kritischen Blick auf die literarischen Quellen weitgehend fehlen lässt und sie mehrheitlich wörtlich liest, ohne die möglichen Intentionen der Autoren hinsichtlich der entsprechenden Passagen zu hinterfragen. Die Gewichtung von deren Aussagekraft - wie im Falle der dynastischen Loyalität etwa insbesondere gegenüber Germanicus und dessen Nachkommen, die er überzeichnet - ist nicht immer nachvollziehbar. ${ }^{119}$ Dieser Umgang mit den Quellen zeigt sich auch im Katalog und ist - neben dem nicht erfüllten Anspruch der Vollständigkeit ${ }^{120}$ - dessen zentraler Schwachpunkt. Jeder erwähnte Auftritt des Volkes wird als faktisches historisches Ereignis gewertet; die Kategorie ,Gewalt‘ (Form: physisch, verbal; Urheber) wird nicht eindeutig gehandhabt. ${ }^{121}$ Vereinzelt wird eine Episode in mehrere zergliedert $^{122}$ - umgekehrt allerdings auch mehrere zu einer zusammengefasst. ${ }^{123}$ Aktionen werden aufgenommen, welche die antiken Autoren nicht etwa der stadtrömischen Plebs zuschreiben, sondern einem Volk anderer geographischer Provenienz oder einer als Masse bezeichneten anderen gesellschaftlichen Gruppierung. ${ }^{124}$ Hinsichtlich der

Manipulation VANDEVOORDE 2015, 982; allgemein zur Zentralität derselben für die Bewertung der politischen Rolle der Plebs ZiMMERMANN 1999, 140f.

118 COURRIER 2014, 642 Anm. 151; 736; 641 Anm. 149 (Demonstrationen: Tac. ann. 14,60,5-61,2); vgl. die im Katalog in der Kategorie ,Spontaneität' von ihm gelegentlich gesetzten Fragezeichen in Klammern - u.a. $888 \mathrm{Nr}$. 243 zu der im Hauptteil noch als spontan deklarierten Aufforderung an Claudius, die Ehe mit Agrippina zu schließen.

${ }^{119}$ Besonders eklatant zeigt sich dies EBD., 729-733; 894 Nr. 256 in seiner Bewertung der Octavia im Kontext der genannten Demonstrationen für sie. Mit der Begründung, dass sich Nero moralisch als Angehöriger der Domus des Germanicus disqualifiziert habe, interpretiert er Octavia als einzig übriggebliebenes Mitglied derselben. Die Aktion der Plebs erklärt er aus dieser Interpretation heraus als Versuch, das Erbe des Germanicus zu retten.

${ }^{120}$ Es fehlen einzelne Parallelstellen: bspw. Suet. Cal. 41,1 zu 884 Nr. 234 (Aktionen im Rahmen der Steuerepisode unter Caligula 40 n. Chr.); Suet. Nero 22,2 zu 890 Nr. 249 (Zuschauer von Neros ersten Auftritten 59 n. Chr.). Es fehlen aber ebenso ganze Episoden: etwa Tac. ann. 2,34,3 zum populus als Zaungast beim Gang des Tiberius vom Palatin zum Forum sowie im Gespräch mit dem Princeps; 3,23,1 zum Mitleid mit Aemilia Lepida und den Verwünschungen gegen Quirinius 20 n. Chr.; Suet. Cal. 2 zur Reaktion des populus auf Pisos Rückkehr 20 n. Chr.; D.C. $58,2,7$ f. (Xiph.) zu Gesandtschaften zu und der Aufstellung von Statuen für Seian 29 n. Chr. Vgl. bereits SMITH 2015.

${ }^{121}$ So z.B. Courrier 2014, 884 Nr. 234 (D.C. 59,28,11 [Xiph.; Exc. Val.]; 59,28,11 [Joann. Antioch.]; J. AJ 19,25f.: Gewaltanwendung durch die Soldaten als gewalttätige Aktion eingestuft); 885 Nr. 237 (Suet. Claud. 12,3: Drohungen gegenüber Militär und Senat angesichts der Befürchtung, Claudius sei einem Anschlag zum Opfer gefallen, als Gewalt kategorisiert).

${ }^{122}$ S. etwa EBD., 877f. Nr. 219 und 220 (Unruhen im Kontext des Sturzes Seians 31 n. Chr.); 880f. Nr. 227 und 882f. Nr. 230; 231 (Opposition gegen Caligula im Kontext von Spielen des Jahres 39 n. Chr.). Bezeichnenderweise decken sich die jeweiligen Stellenangaben größtenteils.

${ }^{123}$ S. etwa EBD., 870 Nr. 208 (Tac. ann. 2,87,1: Forderung der plebs nach Getreide 19 n. Chr.; 2,59,1; Suet. Tib. 52,2; J. Ap. 2,63: Germanicus in Alexandria, Senkung der Getreidepreise, Hungersnot, mit Tacitus wohl ebenfalls 19 n. Chr.); 861f. Nr. 193 (Suet. Aug. 41,2: subventionierter Getreidekauf oder -spenden als häufig ergriffene Maßnahme bei Versorgungsschwierigkeiten, folglich undatiert; 42,3: Ausweisungen von Sklaven, Gladiatoren und Peregrinen, datiert mit D.C. 55,26,1 6 n. Chr.; verworfene Erwägung des Augustus, die frumentatio abzuschaffen); D.C. 55,22,3 (Hungersnot 5 n. Chr., ohne Erwähnung des Volkes); 55,26,3f. (Getreidespende, Hilfe anlässlich eines Brandes 6 n. Chr., ohne Erwähnung des Volkes); 55,27,1-3 (Unruhen, Putschpläne, Spiele, 6 n. Chr.); 55,31,3 (Aufregung angesichts einer Hungersnot und der Kriege, 7 n. Chr.).

${ }^{124}$ S. etwa EBD., 854 Nr. 180 (R. Gest. div. Aug. 10,2: Ernennung des Augustus zum pontifex maximus: ex Italia confluens multitudo); 890 Nr. 248 (Tac. ann. 14,8,1: Reaktionen einer multitudo auf die vermeintliche Ermordung Agrippinas durch Nero nach dem ersten Versuch - diese Menge ist jedoch diejenige Kampaniens; das Volk von Rom, um dessen Zuneigung Nero bis zu seinem Einzug gebangt habe, kommt erst 14,13,1 ins Spiel). - Hinter 
Stellenangaben wird nicht unterschieden zwischen den Kernstellen, die eine Aktion, und den (meist weitaus zahlreicheren) Parallelstellen, die lediglich den Kontext beschreiben. ${ }^{125}$ Damit trägt Courrier zusätzlich einem zentralen Befund zur Plebs nicht Rechnung: der ebenso prekären wie gerade deshalb aufschlussreichen Überlieferungssituation, dass in der Mehrzahl der Fälle selbst diejenigen Aktionen, die für den Kurs der Kaiserzeit wesentlich waren, nur von einem einzigen Autor überliefert und/oder näher dargestellt werden. ${ }^{126}$ Insgesamt ergibt sich eine Verzerrung des Bildes der politischen Aktivität der Plebs, die eine vorbehaltlose Nutzung seines Katalogs im Rahmen zukünftiger Studien nicht zulässt.

Es lässt sich festhalten, dass sich die Forschung seit der Arbeit von Yavetz mit wenigen Ausnahmen einig ist, dass von einer wie auch immer gearteten politischen Bedeutung der stadtrömischen Plebs auszugehen ${ }^{127}$ und diese im recht vagen Rahmen des consensus universorum anzusiedeln ist. Wie diese Bedeutung sich auswirkt und wie hoch die politischen Konsequenzen zu veranschlagen sind, darüber herrscht freilich Dissens. Bedingt ist dieser weniger durch unterschiedliche Quelleninterpretationen, die in etlichen Fällen zur Beantwortung dieser Frage gar nicht als notwendig angesehen werden, als vielmehr durch voneinander abweichende Definitionen von, politisch' sowie durch unterschiedliche Anschauungen des politischen Systems der Kaiserzeit, die den jeweiligen Arbeiten zugrunde liegen. ${ }^{128}$ Insbesondere die Bewertung der Handlungen der Plebs im Sinne einer ,tatsächlichen Einflussnahme ‘ auf den Verlauf der Geschichte ist wenig gewinnbringend und wurde zu Recht mehrheitlich verworfen.

Über die Ergebnisse der zwei Monographien zur Plebs der ersten Forschungsphase und insbesondere Yavetz' Plebs and Princeps aus dem Jahr 1969 ist die Forschung kaum hinausgekommen - trotz des von diesen hinterlassenen Eindrucks der Blässe der Plebs, die aus dem Fokus auf die ,Geberseite' resultiert, und obwohl der Untersuchungszeitraum durch nachfolgende Studien (hierbei ist insbesondere Courrier hervorzuheben) erweitert wurde. Bezeichnend ist, dass noch in einem 2012 erschienenen Sammelband zur Politische[n] Kommunikation und öffentliche[n] Meinung in der antiken Welt die Relevanz der kaiserzeitlichen Plebs von der Herausgeberin, Christina Kuhn, zwar hervorgehoben wird, ein eigener Beitrag dazu jedoch fehlt: Verwiesen wird lediglich auf Yavetz und Gilbert. ${ }^{129}$ Nur selten wurden bisher auch Forschungsdesiderate formuliert: Das mag daran liegen, dass die Plebs bis heute als

anderen Erwähnungen des Volkes in den Quellen, wie den Wahlempfehlungen an das Volk 8 n. Chr. (D.C.

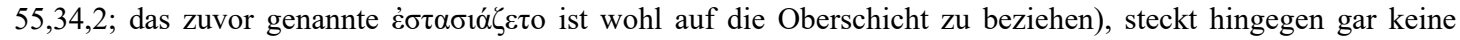
(Re-)Aktion des Volkes: s. jedoch EBD., 862 Nr. 194. Die hier als „peu intelligible“ bezeichnete Unterscheidung zwischen $\pi \lambda \tilde{\eta} \theta$ o $\varsigma$ und $\delta \tilde{\eta} \mu \circ \varsigma$ bezieht sich nicht auf das Volk als Gruppierung, sondern auf die unterschiedlichen Typen von Volksversammlungen (concilium plebis, comitia) und begegnet bei Dio regelmäßig: vgl. RICH 1990, 155 zu D.C. 53,21,6; FreYBuRGER-GALLAND 1997, 84-86; DE BLOIS 1997, 2656.

${ }^{125}$ S. etwa COURRIER 2014, 860f. Nr. 191 (Forderung nach der Rückkehr Iulias); 861f. Nr. 193 (Versorgungskrisen mit Unruhen 6 n. Chr. sowie 7/8 n. Chr.; wie Anm. 123); vgl. 854 Nr. 180 und 890 Nr. 248 (beide wie Anm. 124).

${ }^{126}$ Das trifft etwa auf die Antragung der Diktatur und der cura annonae an Augustus $22 \mathrm{v}$. Chr. zu. Allein Dio beschreibt die Aktion der Plebs ausführlich, während nicht nur Augustus selbst, sondern ebenso Sueton und Velleius lediglich auf die Beteiligung des populus an der Antragung verweisen: D.C. 54,1,2-4; Suet. Aug. 52; R. Gest. div. Aug. 5,1f.; Vell. 2,89,5.

127 Die Behauptung von SünSKes ThOMPSON 1993, 2, dass die Ansicht Veynes (s. oben) die communis opinio widerspiegle, ist nicht nachvollziehbar.

${ }^{128}$ Dies reflektiert den diesbezüglichen Dissens auch in der Politikwissenschaft: s. etwa MEYER 201033, 37-39, der die „verwirrende Fülle von Definitionen des Politischen“ (37) auf die Auswahl zurückführt, „welches ihrer Elemente oder welche ihrer in der Definition selbst unausgesprochenen Voraussetzungen jeweils in besonderer Weise herausgehoben und beleuchtet werden" (39).

${ }^{129}$ KUHN 2012, 18-20 mit Anm. 30. 
schwieriges Sujet gilt, über das die ,Oberschichtenliteratur' kaum Aussagen zulasse. Wo Desiderate genannt wurden, betreffen sie die Frage nach Mechanismen und möglichen Interaktionen zwischen den einzelnen Gruppierungen, mit denen die Beurteilung der politischen Bedeutung der stadtrömischen Plebs steht und fällt. Dass sie kaum beantwortet wurde, liegt an zwei grundsätzlichen Problemen des Umgangs mit den literarischen Quellenbelegen zur Plebs.

Das erste Problem betrifft die allzu große Bereitschaft, den einzelnen Quellenaussagen zur Plebs unkritisch Glauben zu schenken, beinahe getreu dem Motto, dass das Wenige, das die antiken Autoren zu ihr berichten, doch wenigstens wahr zu sein habe - eine Tendenz, vor der Benjamin Kelly in einer Studie zu Aufständen von Unterschichten im römischen Reich aus dem Jahr 2007 warnt. ${ }^{130}$ Obwohl es communis opinio ist, dass die antiken Historiographen und Biographen auch Literaten sind, wird in Bezug auf das Sujet ,Plebs ' daran nur von vereinzelten Althistorikern festgehalten, deren Forschungsinteresse zudem - und wohl bezeichnenderweise - nicht zentral auf dem stadtrömischen Volk liegt. ${ }^{131}$ Wo dies in den einschlägigen Studien erwähnt wird, geschieht es einmalig und ohne Analyse. Dabei werden Belege entweder aus einem Zeitraum, der in sicherer Entfernung vom untersuchten liegt, als Exempel angeführt (Yavetz) oder aber, quasi als nachträglicher Gedankenblitz, im letzten (!) Absatz des Fazits (Sünskes Thompson) erwähnt. ${ }^{132}$

Das zweite, ähnlich banale, aber grundsätzliche Problem hängt damit zusammen. Es besteht in der isolierten Betrachtung jener Stellen, in denen die Plebs erwähnt wird. Das, was die neuere Forschung an der älteren hinsichtlich des Umgangs mit Iuvenals einprägsamem Schlagwort von panis et circenses kritisiert, nämlich die weitgehende Eins-zu-Eins-Übernahme ohne Beachtung des Kontextes, das praktiziert sie in Bezug auf zahlreiche weitere Passagen zur Plebs selbst. Episoden werden allzu oft nur referiert und aneinandergereiht statt analysiert. Kaum wird auf die textuelle und/oder historische Einbindung von Belegen zur Plebs in übergeordnete Sachverhalte geachtet. Noch weniger wird allerdings der Duktus des Textes selbst berücksichtigt, also einer möglichen literarischen Absicht hinter der Einbeziehung der Plebs in die Darstellung Rechnung getragen. Sie muss jedoch der Ausgangspunkt einer jeden Untersuchung über die Plebs wie auch über andere ,Randfiguren' der Gesellschaft sein, um (auch) zu differenzierten historischen Erkenntnissen zu gelangen. Dass dies möglich und gleichzeitig die historische Aussagekraft der Plebs als rhetorischen Elements der antiken politischen Literatur - dann freilich in Bezug auf die Perspektive der Autoren - nicht zu unterschätzen ist, das legt die Verfasserin in der demnächst erscheinenden Monographie Die politische Rolle der stadtrömischen Plebs in der Kaiserzeit dar. ${ }^{133}$

\footnotetext{
${ }^{130}$ KeLLY 2007, 151f. In diesem Aufsatz fasst Kelly zentrale Ergebnisse seiner unpublizierten Dissertation The Repression of Violence in the Roman Principate (Oxford 2003) zusammen. - Die diametral entgegengesetzte Position, die neben Kelly (s. insbes. 151-156) etwa BLEICKEN 1998, 793 vertritt, nämlich den Quellen jegliche Aussagekraft bezüglich der Plebs abzusprechen, ist freilich ebenso problematisch.

${ }^{131}$ So KNEPPE 1994, insbes. 92; 329; ZIMMERMANN 1999, 133.

${ }^{132}$ YAvetz 1969a, 5 zu D.C. 74(73),13,2-5 (Xiph.) und 76(75),4,2-6 (Xiph.); SüNSKes ThOMPSON 1993, 73f.

${ }^{133}$ KRÖSS 2017.
} 


\section{Literaturverzeichnis}

АвBотт 1911

AFrICA 1965

AFRICA 1971

ALFÖLDY $2011^{4}$

ARENA 2007

BADIAN 1970

BALDWIN 1972

BALSDON 1969a

BALSDON 1969b

BALTRUSCH 1994

BANDELLI 1969

BERNARDI 1970

BLEICKEN 1998

BOLLINGER 1969

FRANK F. ABBOTT: Studies of Roman Life and Literature, New York 1911 (ND 1965)

Thomas W. Africa: Rome of the Caesars, New York/London/Sydney 1965

DERS.: Urban Violence in Imperial Rome, Journ. of Interdisciplinary Hist. 2/1971, 3-21

GÉZA ALFÖLDY: Römische Sozialgeschichte, Stuttgart $2011^{4}$

PATRIZIA ARENA: Turba quae in foro litigat, spectat in theatris (Sen. Cons. ad Marc. 11, 2). Osservazioni sull'utilizzo del sostantivo turba in Seneca, Tacito e Suetonio, in: Elio Lo Cascio/Giovanna D. Merola (Hrsg.): Forme di aggregazione nel mondo romano, Bari 2007, 13-30

ERnst Badian: Rez. Zvi Yavetz: Plebs and Princeps, New Brunswick 1969, Phoenix 24/1970, 93-94

BARRY BALDWIN: Rulers and Ruled at Rome A.D. 14-192, AncSoc 3/1972, 149-163

JOHN P.V.D. BALSDON: Life and Leisure in Ancient Rome, London 1969

Ders.: Mob and Master. Rez. Zvi Yavetz: Plebs and Princeps, New Brunswick 1969, TLS 68/1969, 780

ERnST BAltrusch: Rez. EgOn Flaig: Den Kaiser herausfordern. Die Usurpation im Römischen Reich, Frankfurt a. M./New York 1992, HZ 259/1994, 454456

Gino BAndelli: Rez. Zvi Yavetz: Plebs and Princeps, New Brunswick 1969, P\&I 11/1969, 353356

Aurelio Bernardi: Rez. ZVi YaVetz: Plebs and Princeps, New Brunswick 1969, Athenaeum 48/1970, 175-179

JOCHEN Bleicken: Rez. Zvi YAVETZ: Plebs and Princeps, New Brunswick 1969 (1970), in: DERS.: Gesammelte Schriften II, Stuttgart 1998, 792-795

TraugotT Bollinger: Theatralis licentia. Die Publikumsdemonstrationen an den öffentlichen Spielen im Rom der frühen Kaiserzeit und ihre Bedeutung im politischen Leben, Winterthur 1969 
BRAUNERT 1980

CAMPBELl 1995

CARCOPINO $1992^{4}$

CAUER 1899

COURRIER 2014

DE BLOIS 1997

DEFOSSE 1969

DEININGER 1979

DIESNER 1973

FLAIG 1992

FLAIG 1994

FLAIG 1998

FRASCHETTI $2005^{2}$
HORST BRAUNERT: Großstadt und Großstadtprobleme im Altertum, in: DeRS.: Politik, Recht und Gesellschaft in der griechisch-römischen Antike. Gesammelte Aufsätze und Reden, Stuttgart 1980, 11-28

BRIAN CAMPBEll: Rez. JULIA SÜNSKES THOMPSON: Demonstrative Legitimation der Kaiserherrschaft im Epochenvergleich. Zur politischen Macht des stadtrömischen Volkes, Stuttgart 1993, CR 45/1995, 348349

JÉRÔME CARCOPINO: Rom. Leben und Kultur in der Kaiserzeit, Stuttgart $1992^{4}$

FRIEDRICH CAUER: Die Stellung der arbeitenden Klassen in Hellas und Rom, Neue Jahrbücher für das klassische Altertum, Geschichte und deutsche Literatur und für Pädagogik 3/1899, 686-702

CYRIL COURRIER: La plèbe de Rome et sa culture (fin du $\mathrm{II}^{\mathrm{e}}$ siècle av. J.-C. - fin du $\mathrm{I}^{\mathrm{er}}$ siècle ap. J.-C.), Rome 2014

LUKAS DE BLOIS: Volk und Soldaten bei Cassius Dio, in: ANRW II 34,3 (1997), 2650-2676

Pol Defosse: Rez. Zvi Yavetz: Plebs and Princeps, New Brunswick 1969, Latomus 28/1969, 799-800

JÜRgEn DeININGER: Brot und Spiele. Tacitus und die Entpolitisierung der plebs urbana, Gymnasium 86/1979, 278-303

HANS-JoAChIM Diesner: Rez. ZVI YAVETZ: Plebs and Princeps, New Brunswick 1969, AAHG 26/1973, 88-90

EgON FLAIG: Den Kaiser herausfordern. Die Usurpation im Römischen Reich, Frankfurt a.M./New York 1992

DERS.: Das politische System des Principats, in: JoCHEN MARTIN (Hrsg.): Das alte Rom. Geschichte und Kultur des Imperium Romanum, München 1994, $117-132$

DeRS.: Rez. JULIA SÜNSKES ThOMPSON: Demonstrative Legitimation der Kaiserherrschaft im Epochenvergleich. Zur politischen Macht des stadtrömischen Volkes, Stuttgart 1993, Gnomon 70/1998, 135-139

Augusto Fraschetti: Roma e il principe, Bari $2005^{2}$

http://www.fera-journal.eu 
Freyburger-Galland 1997 Marie-Laure Freyburger-Galland: Aspects du vocabulaire politique et institutionnel de Dion Cassius, Paris 1997

FREZZA 1969

Paulo Frezza: Rez. Zvi Yavetz: Plebs and Prin-

FRIEDLÄNDER $1922^{10}$ ceps, New Brunswick 1969, SDHI 35/1969, 464-465

GABBA 1969

LUDWIG FRIEDLÄNDER: Darstellungen aus der Sittengeschichte Roms. In der Zeit von Augustus bis zum Ausgang der Antonine, 4 Bd., 10. Auflage besorgt von Georg Wissowa, Leipzig $1922^{10}$ (ND 1979)

EMilio GabBa: Rez. ZVI YAVEtz: Plebs and Princeps, New Brunswick 1969, RFIC 67/1969, 477479

GAGE $1971^{2}$

GARNSEY/SALLER 1987

GAVAZZI 1977

JEAN GAGE: Les classes sociales dans l'empire Romain, Paris $1971^{2}$

Peter GARnSEy/Richard SALler: The Roman Empire. Economy, Society and Culture, London 1987

LEONARDO GAVAZZI: Plebs e princeps, NRS 61/1977, $1-9$

GILBERT 1976

Rolf GILBERT: Die Beziehungen zwischen Princeps und stadtrömischer Plebs im frühen Principat, Bochum 1976

GRIFFIN 1991

MiRIAM T. GRIFFIN: Urbs Roma, Plebs and Princeps, in: ALEXANDER LOVEDAY (Hrsg.): Images of Empire, Sheffield 1991, 19-46

GROOT 2008

HELEEN GROOT: Zur Bedeutung der öffentlichen Spiele bei Tacitus, Sueton und Cassius Dio. Überlegungen zur Selbstbeschreibung der römischen $\mathrm{Ge}$ sellschaft, Berlin 2008

GRUEN 1970

Erich S. Gruen: Rez. Zvi Yavetz: Plebs and Princeps, New Brunswick 1969, AJPh 91/1970, 487-489

HAHN 1969

ISTVÁN HAHN: Zur politischen Rolle der stadtrömischen Plebs unter dem Principat, in: VESELIN BEŠEVLiev/Wolfgang Seyfarth (Hrsg.): Die Rolle der Plebs im Spätrömischen Reich. Görlitzer EireneTagung 10.-14.10.1967, Bd. 2, Berlin 1969, 39-54

HELLEGOUARC'H 1971

Joseph Hellegouarc'H: Rez. Zvi Yavetz: Plebs and Princeps, New Brunswick 1969, RPh 45/1971, 182-184

HELLEGOUARC'H $1972^{2}$

DERS.: Le vocabulaire latin des relations et des partis politiques sous la république, Paris $1972^{2}$

HOFFMANN 1938

Wilhelm HofFMANN: Die römische Plebs, NJAB 1/1938, 82-98 
HOFFMANN 1951

HoHL 1933

HORSTKOTTE 1995

HURLET 1997

HURLET 2009

HURLEY 1993

INSTINSKY 1940

JAL 1969

JONES 1971

KAHRSTEDT $1958^{2}$

KELLY 2007

KIENAST $2009^{4}$

KNEPPE 1994

KRÖSS 2017

KRÖSS (im Erscheinen)
DERS.: s. v. Plebs, in: RE 21,1 (1951), 73-103

ERNST HOHL: Wann hat Tiberius das Prinzipat übernommen?, Hermes 68/1933, 106-115

Hermann HorstKotte: Rez. EgOn Flaig: Den Kaiser herausfordern. Usurpation im Römischen Reich, Frankfurt a.M./New York 1992, Klio 77/1995, 517519

FREDERIC HURLET: Les Collègues du prince sous Auguste et Tibère. De la légalité républicaine à la légitimité dynastique, Rome 1997

DERS.: L'aristocratie augustéenne et la res publica restituta, in: DERS./BERNARD MINEO (Hrsg.): Le Principat d'Auguste. Réalités et représentations du pouvoir. Autour de la Res publica restituta, Rennes 2009, 73-99

Donna W. Hurley: An Historical and Historiographical Commentary on Suetonius' Life of C. Caligula, Atlanta, Georgia 1993

ULRICH INSTINSKY: Consensus universorum, Hermes 75/1940, 265-278

Paul Jal: Rez. Zvi Yavetz: Plebs and Princeps, New Brunswick 1969, REA 71/1969, 553-555

ARnold H.M. Jones: Rez. Zvi Yavetz: Plebs and Princeps, New Brunswick 1969, EHR 86/1971, 378379

ULRICH KAHRSTEDT: Kulturgeschichte der römischen Kaiserzeit, Bern $1958^{2}$

BenJAMIN KeLLY: Riot Control and Imperial Ideology in the Roman Empire, Phoenix 61/2007, 150-176

DietMar KIENAST: Augustus. Prinzeps und Monarch, Darmstadt $2009^{4}$

AlfRED KNEPPE: Metus temporum. Zur Bedeutung von Angst in Politik und Gesellschaft der römischen Kaiserzeit des 1. und 2. Jhdts. n. Chr., Stuttgart 1994

KATJA KRÖSS: Die politische Rolle der stadtrömischen Plebs in der Kaiserzeit, Leiden/Boston 2017 (im Druck)

DIES.: Die stadtrömische Plebs in den zeitgeschichtlichen Büchern Cassius Dios, in: ElKe HARTMAnN/Sven Page/Anabelle ThuRn (Hrsg.): Moral

http://www.fera-journal.eu 
KUHN 2012

KUNKEL 1961

LASER 1997

LENDON 1997

LEPPIN 1992

LEVICK 2010

MACMULLEN 1966

MARQUARDT $1886^{2}$

MARTIN 1991

MAXEY 1938

MEYER $2010^{3}$

MiLlaR $1992^{2}$

MOMMSEN $1882^{7}$

NIPPEL 1988 als Kapital im antiken Athen und Rom, Stuttgart (im Erscheinen)

CHRISTINA KUHN: Politische Kommunikation und öffentliche Meinung in der antiken Welt. Einleitende Bemerkungen, in: Dies. (Hrsg.): Politische Kommunikation und öffentliche Meinung in der antiken Welt, Stuttgart 2012, 11-30

WOLFGANG KunKEL: Über das Wesen des augusteischen Prinzipats, Gymnasium 68/1961, 353370

GÜNTER LASER: Populo et scaenae serviendum est, Trier 1997

JON E. LENDON: Empire of Honour. The Art of Government in the Roman World, Oxford 1997

HARTMUT LEPPIN: Histrionen. Untersuchungen zur sozialen Stellung von Bühnenkünstlern im Westen des Römischen Reiches zur Zeit der Republik und des Principats, Bonn 1992

BARBARA M. LEVICK: Augustus. Image and Substance, Harlow/London/New York 2010

RAMSAY MACMULLEN: Enemies of the Roman Order. Treason, Unrest, and Alienation in the Empire, Cambridge, Massachusetts 1966

JOACHIM MARQUARDT: Privatleben der Römer. 2 Bd., Darmstadt $1886^{2}$

JEAN-PIERRE MARTIN: Le pouvoir impérial romain: sa spécificité idéologique, in: o. Hrsg.: L'idéologie du pouvoir monarchique dans l'Antiquité. Actes du colloque de la Société des Professeurs d'Histoire Ancienne de 1'Université tenu à Lyon et Vienne les 26 28 juin 1989, Paris 1991, 77-89

MiMA MAXEY: Occupations of the Lower Classes in Roman Society, Chicago 1938

Thomas Meyer: Was ist Politik?, Opladen $2010^{3}$

Fergus MillaR: The Emperor in the Roman World (31 BC - AD 337), London $1992^{2}$

THEOdOR MOMMSEN: Römische Geschichte. Dritter Band: Von Sullas Tode bis zur Schlacht von Thapsus, Berlin $1882^{7}$

WILFRIED NIPPEL: Aufruhr und ,Polizei' in der römischen Republik, Stuttgart 1988

http://www.fera-journal.eu 
NIPPEL 1995

Oost 1971

PEKÁRY 1987

PETIT 1970

REESE 2004

RICH 1990

RICHARD 1970

ROSTOVTZEFF $1957^{2}$

SALLER 1990 [2000-01]

SCARBOROUGH 1971

SILLETT 2016

SMITH 1987

SMITH 2015

SÜNSKES THOMPSON 1990
Ders.: Public Order in Ancient Rome, Cambridge 1995

Stewart I. Oost: Rez. Zvi Yavetz: Plebs and Princeps, New Brunswick 1969, CPh 66/1971, 137-138

THOMAs PEKÁRY: Seditio. Unruhen und Revolten im römischen Reich von Augustus bis Commodus, AncSoc 18/1987, 133-150

Paul Petit: Rez. Zvi Yavetz: Plebs and Princeps, New Brunswick 1969, AC 39/1970, 313-314

ALFRED REESE: Die Bürger und ihr Kaiser. Die plebs urbana zwischen Republik und Prinzipat, Bochum 2004 (Onlinepublikation, zuletzt geprüft am 15.12.2016: $\quad$ http://www-brs.ub.ruhr-unibochum.de/netahtml/HSS/Diss/ReeseAlfred/diss.pdf)

JoHN W. Rich: Cassius Dio. The Augustan Settlement (Roman History 53-55,9), Warminster 1990

JEAN-Claude Richard: Rez. Zvi Yavetz: Plebs and Princeps, New Brunswick 1969, REL 48/1970, 627629

Michael I. RostovtzefF: The Social and Economic History of the Roman Empire, 2 Bd., Oxford $1957^{2}$

RICHARD SALLER: Domitian and his Successors. Methodological Traps in Assessing Emperors, AJAH 15/1990 [2000-01], 4-18

John Scarborough: Rez. Zvi Yavetz: Plebs and Princeps, New Brunswick 1969, CJ 66/1971, 264-266

ANDREW J. SILLETT: The Culture of the Roman Plebs. Rez. Cyril Courrier: La plèbe de Rome et sa culture (fin du $\mathrm{II}^{\mathrm{e}}$ siècle av. J.-C. - fin du $\mathrm{I}^{\mathrm{er}}$ siècle ap. J.-C.), Rome 2014, CR 66/2016, 208-210

RONALD R.R. SMITH: The Imperial Reliefs from the Sebasteion at Aphrodisias, JRS 77/1987, 88-138

Christopher SMith: Rez. CYril Courrier: La plèbe de Rome et sa culture (fin du $\mathrm{II}^{\mathrm{e}}$ siècle av. J.-C. - fin du ${ }^{\text {er }}$ siècle ap. J.-C.), Rome 2014, BMCR 2015.08.09

JULIA SÜNSKES THOMPSON: Aufstände und Protestaktionen im Imperium Romanum. Die severischen Kaiser im Spannungsfeld innenpolitischer Konflikte, Bonn 1990 
SÜNSKES THOMPSON 1993

SYME 1939

URBAN 1995

VAN BERCHEM 1939

VANDEVOORDE 2015

VEYNE 1976

VEYNE 2005

VIRLOUVET 1985

VON PREMERSTEIN 1937

WEBER 1936

WICKERT 1970

WITSCHEL 2006

YAVETZ 1958

YAVETZ 1965a

YAVETZ 1965b

YAVETZ 1969a
DIES.: Demonstrative Legitimation der Kaiserherrschaft im Epochenvergleich. Zur politischen Macht des stadtrömischen Volkes, Stuttgart 1993

RonALD SyME: The Roman Revolution, Oxford 1939

RAlF URBAn: Rez. EgON Flaig: Den Kaiser herausfordern. Usurpation im Römischen Reich, Frankfurt a.M./New York 1992, BJ 195/1995, 691-700

DENIS VAN BERCHEM: Les distributions de blé et d'argent à la plèbe romaine sous l'empire, Genève 1939 (ND 1975)

LindSEY VANDEVOORDE: Rez. CyRIL COURRIER: La plèbe de Rome et sa culture (fin du II ${ }^{\mathrm{e}}$ siècle av. J.-C. - fin du I ${ }^{\mathrm{er}}$ siècle ap. J.-C.), Rome 2014, Annales (HSS) 70/2015, 981-983

Paul Veyne: Le pain et le cirque. Sociologie historique d'un pluralisme politique, Paris 1976

DERS.: Qu'était-ce qu'un empereur romain?, in: DERS.: L'Empire gréco-romain, Paris 2005, 15-78

CATHERINE VIRLOUVET: Famines et émeutes à Rome des origines de la République à la mort de Néron, Rome 1985

Anton Von Premerstein: Vom Werden und Wesen des Prinzipats, München 1937

Wilhelm Weber: Princeps. Studien zur Geschichte des Augustus I, Stuttgart 1936

LOTHAR Wickert: Rez. Zvi YAVETZ: Plebs and Princeps, New Brunswick 1969, HZ 211/1970, 652653

CHRISTIAN WiTSCHEL: Verrückte Kaiser? Zur Selbststilisierung und Außenwahrnehmung nonkonformer Herrscherfiguren in der römischen Kaiserzeit, in: CHRISTIAN RonNING (Hrsg.): Einblicke in die Antike. Orte - Praktiken - Strukturen, München 2006, 87125

ZVI YAVETZ: The Living Conditions of the Urban Plebs, Latomus 17/1958, 500-517

DERS.: Levitas popularis, A\&R 10/1965, 97-110

DERS.: Plebs sordida, Athenaeum 43/1965, 295-311

DERS.: Plebs and Princeps, New Brunswick 1969 (ND 1988 mit neuem Vorwort) 
YAVETZ 1969b

YAVETZ 1979

YAVETZ 1987

ZIMMERMANN 1999
DERS.: Vitellius and the ,Fickleness of the Mob', Historia 18/1969, 557-568

DERS.: Caesar in der öffentlichen Meinung, Düsseldorf 1979

DERS.: The Urban Plebs in the Days of the Flavians, Nerva and Trajan, in: AdALBERTo GIOVANNINI/KURT A. RaAflaub (Hrsg.): Opposition et résistances à l'empire d'Auguste à Trajan, Genève 1987, 135-181

MARTIN ZIMMERMANN: Herodians Konstruktion der Geschichte und sein Blick auf das stadtrömische Volk, in: DERS. (Hrsg.): Geschichtsschreibung und politischer Wandel im 3. Jh. n. Chr. Kolloquium zu Ehren von Karl-Ernst Petzold (Juni 1998) anlässlich seines 80. Geburtstags, Stuttgart 1999, 119-143

\section{Kontakt zur Autorin:}

Katja Kröss

Ludwig-Maximilians-Universität

Graduiertenschule Distant Worlds

Geschwister-Scholl-Platz 1

80539 München

Email:katja.kroess@1mu.de 